HUTP-93/A040

\title{
Quantum cohomology of partial flag manifolds $F_{n_{1} \cdots n_{k}}$
}

\author{
Alexander Astashkevich \\ Department of Mathematics \\ M.I.T. \\ Cambridge, MA 02139 \\ and \\ Vladimir Sadov \\ Lyman Laboratory of Physics \\ Harvard University \\ Cambridge, MA 02138 \\ and
}

L.D. Landau Institute for Theoretical Physics, Moscow

We compute the quantum cohomology rings of the partial flag manifolds $F_{n_{1} \cdots n_{k}}=$ $U(n) /\left(U\left(n_{1}\right) \times \cdots \times U\left(n_{k}\right)\right)$. The inductive computation uses the idea of Givental and Kim [1]. Also we define a notion of the vertical quantum cohomology ring of the algebraic bundle. For the flag bundle $F_{n_{1} \cdots n_{k}}(E)$ associated with the vector bundle $E$ this ring is found.

$12 / 93$ 


\section{Introduction and summary.}

The quantum cohomology rings are only known for some classes of varieties. For Calabi-Yau manifolds one [2] can transform the problem to the "mirror dual" one which deals with variations of the Hodge Structure. The latter is "in principle" solvable, although the real computations in terms of Picard-Fuchs equations may be pretty hard. All known examples describe one- or two- parametric deformations of the classical cohomology rings (see for example [3] [4] [5]).

The ring $Q H^{*}\left(\mathbf{P}^{n}\right)=\mathbf{C}[x] /\left(x^{n+1}-q\right)$ for the projective spaces has been known since long ago in physics [6] and mathematics (symplectic Floer theory [7]). More generally, one can construct the moduli spaces of rational curves for the toric varieties [8]. Recently Batyrev [9] has conjectured a general formula for the quantum ring in that case. From the physical point of view Batyrev's result can be obtained using the Hamiltonian reduction of the linear $\sigma$-model by the real torus [10].

Another example where the hamiltonian reduction of the linear $\sigma$-model (by $U(n)$ ) does work [11], [10] is the Grassmanian $G r(n, m)=U(n) /(U(n-m) \times U(m))$, because this manifold can be presented as $G r(n, m)=\mathbf{C}^{n m} / / U(m)$. The relations in $Q H^{*}(G r(n, m))$ were discussed in many physical papers [12] [13] [14] [15] [16] [17]. They are proven mathematically [18] for the Grassmanians of 2-planes.

Recently Givental and Kim [1] have computed the quantum cohomology ring of the complete flag manifold $F_{n}=U(n) /(U(1))^{\times n}$. The main idea of their paper was to use the functoriality properties of the equivariant quantum cohomology. Here we extend the arguments of [1] to cover all partial flag manifolds $F_{n_{1} \cdots n_{k}}=U(n) /\left(U\left(n_{1}\right) \times \cdots \times U\left(n_{k}\right)\right)$ "interpolating" between Grassmanians and complete flag manifolds.

In general, the quantum cohomology is rather difficult to compute. An obstacle for developing any efficient computational technique is our lack of understanding of its functoriality properties. In the classical Algebraic Topology, whenever there is a map $i: X \rightarrow Y$ between two manifolds, there is a pullback morphism $i^{*}$ of their cohomology rings such that $i^{*} H^{*}(Y)$ - a pullback of cohomology of $Y$ - is a subring in $H^{*}(X)$.

For the quantum cohomology rings, we know no analogs of the pullback morphism. As an illustration, let us consider a simple example. Take a map $i: \mathbf{P}^{1} \rightarrow \mathbf{P}^{2}$ of degree one, then cohomology of $\mathbf{P}^{1}$ is generated by the pullback $x=i^{*} y$ of the Kähler class $y$ of $\mathbf{P}^{2}$; this is a particular case of functoriality with respect to the maps between manifolds. Now if we go to the quantum cohomology rings, we see there is a relation $x^{2}=1$ in $Q H^{*}\left(\mathbf{P}^{1}\right)$ 
and another one $y^{3}=1$ in $H^{*}\left(\mathbf{P}^{2}\right)$. There are no non-trivial morphisms between such rings. Here, as in general, it is impossible to define a meaningful pullback for the quantum cohomology.

There is a natural object defined for the algebraic bundles $\pi: X \rightarrow B$ with fiber $F$ a Kähler manifold. We call it the vertical quantum cohomology $\operatorname{ring} Q H_{V}^{*}(X, B, F)$. Its classical limit coincides with the cohomology $\operatorname{ring} H^{*}(X)$ of the total space. The quantum multiplication in $Q H_{V}^{*}(X, B, F)$ is a deformation of multiplication in $H^{*}(X)$ by means of the vertical rational curves $\Sigma$ in $X$, i. e. such that $\pi(\Sigma)=\{p t\}$. The $\operatorname{ring} Q H_{V}^{*}(X, B, F)$ contains $H^{*}(B)$ as a subring and can be considered as a $H^{*}(B)$-algebra. The vertical cohomology is functorial with respect to the base change morphisms $B \rightarrow B^{\prime}$.

A paper has the following structure:

The second section both introduces the basic notions and outlines the main results of the whole paper. In particular, the ring $Q H^{*}\left(F_{n_{1} \cdots n_{k}}\right)$ is described in the Section 2.3 The section is written using in the physical language and is primarily intended for a physicist reader.

The third section discusses essentially the same topics as the first one. More mathematically oriented reader will find there the formal definitions and properties of the objects used in course of subsequent computations.

In the fourth section we discuss the equivariant quantum cohomology, first defined in [1]. In Sec. 4 we give another definition, of more algebraic nature.

The fifth section deals with the computation of the ring $Q H^{*}\left(F_{n_{1} \cdots n_{k}}\right)$. This inductive computation is based on the ideas of [1]. The base of induction is provided by the known answer for the quantum cohomology of Grassmanians $U(n) /\left(U\left(n_{1}\right) \times U\left(n_{2}\right)\right)$. The latter is discussed in many papers [12] - [17] which we summarize in Sec. 5.2. A step of induction uses the functoriality properties of the equivariant quantum cohomology discussed in Sec. 4 .

Acknowledgements. A. A. is very grateful to R. Bezrukavnikov, D. Kaledin, B. Kostant, M. Verbitsky and D. Vogan for valuable discussions. V. S. would like to thank M. Bershadsky and C. Vafa for the helpful discussions and support. We also acknowledge the interesting discussions with S. Piunikhin. Both of us are grateful to Michael Bershadsky for hospitality during the period the major part of this work was being written.

Research of V. S. was supported in part by the Packard Foundation and by NSF grant PHY-87-14654 


\section{A physical introduction to vertical quantum cohomology.}

\subsection{Quantum cohomology}

Quantum cohomology rings are interesting from both the physical and the mathematical points of view. A physicist would say they compute the topological correlation functions in a 2-d $N=2$ supersymmetric $\sigma$-model with a Kähler manifold $X$ as a target space.

The (one-loop) beta-function of the $\sigma$-model is proportional to the Ricci tensor $R_{i \bar{j}}$; its cohomological class represents the first Chern class $c_{1}(X)$ of the tangent bundle $T_{*} X$. If $c_{1}(X)>0$, the renormalization group flow has a stable $U V$ fixed point (large volume limit) 19] where the metrics $G_{i \bar{j}}$ becomes (cohomologicaly) the infinite volume Kähler-Einstein metrics and the $\sigma$-model becomes (asymptotically) free; here the positivity of the first Chern class means that $c_{1}(X)$ belongs to the Kähler cone in $H^{2}(X)$.

An important class of observables in $N=2 \sigma$-models consists of chiral operators [20], which are in one-to-one correspondence with the de Rham cohomology classes of the target space $X$. These operators form a closed subtheory; the correlation functions in this subtheory are called topological correlation functions. In the large volume limit the topological correlation functions are given by the intersection numbers in the cohomology ring $H^{*}(X)$. The nonrenormalization theorems on $N=2$ supersymmetric theories imply that away from the $U V$ fixed point the only possible corrections to these correlation functions are instanton (semi-classical) corrections.

The instantons are simply the holomorphic maps from the world sheet into the target space; the usual fermionic number anomaly argument shows that in the generic situation only isolated maps are to be counted. The instanton sum goes over all possible homological types of these maps. Every summand of a given homological type has an exponential factor $\exp (-A)$ where $A$ is an area of the worldsheet in the pullback metricst, and a pre-exponential factor proportional to the number of the isolated instantons.

The topological correlation functions can be exactly computed in terms of the topological $\sigma$-model [21] 222] [23], related to the original $N=2 \sigma$-model by what is called in [22]a topological $A$-twist. The fundamental fields of the model are

Bosons : world sheet scalars $X^{i}$ - coordinates in the target space

world sheet $1-$ form $F_{\alpha}^{i}$ - target space vector

Fermions : world sheet scalar $\chi^{i}-$ target space vector

world sheet $1-$ form $\rho_{\alpha}^{i}-$ target space vector.

1 This area depends only on the homological type of the holomorphic map. 
The field $F_{\alpha}^{i}$ is what is called the auxiliary field. Both $\rho_{\alpha}^{i}$ and $F_{\alpha}^{i}$ satisfy a self-duality constraint

$$
\begin{aligned}
& \rho_{\alpha}^{i}=i \epsilon_{\alpha}{ }^{\beta} J^{i}{ }_{j} \rho_{\beta}^{j} \\
& F_{\alpha}^{i}=\epsilon_{\alpha}^{\beta} J^{i}{ }_{j} F_{\beta}^{j}
\end{aligned}
$$

The only physical operators in the topological model are the chiral operators of the $N=2$ model. They represent the nontrivial cohomology classes of the BRST operator Q which acts on the multiplet (2.1)as follows

$$
\begin{aligned}
& {\left[Q, X^{i}\right]=i \chi^{i}} \\
& \left\{Q, \chi^{i}\right\}=0 \\
& \left\{Q, \rho_{\alpha}^{i}\right\}=\partial_{\alpha} X^{i}+\epsilon_{\alpha}^{\beta} J_{j}^{i} \partial_{\beta} X^{j}-i \Gamma_{j k}^{i} \chi^{j} \rho_{\alpha}^{k}
\end{aligned}
$$

The physical operators are in one-to-one correspondence with the de Rham cohomology classes of $X$ : given that a differential form $\omega=\omega_{i_{1} \ldots i_{k}}(X) d X^{i_{1}} \ldots d X^{i_{k}}$ represents a class $[\omega] \in H^{k}(X)$ of de Rham cohomology, an operator

$$
\mathcal{O}_{\omega}=\omega_{i_{1} \ldots i_{k}}(X(z, \bar{z})) \chi^{i_{1}}(z, \bar{z}) \ldots \chi^{i_{k}}(z, \bar{z})
$$

represents a class of BRST cohomology.

Often it is more convenient to consider the operator product algebra of the chiral states, a chiral ring or a quantum cohomology ring, instead of the correlation functions. If $\left\{x_{i}\right\}$ is a basis of chiral states, then the ring structure can be written in terms of the three-point correlation functions on sphere as follows:

$$
a \cdot b=\left\langle a b x^{i}\right\rangle x_{i}
$$

where $\left\{x^{i}\right\}$ is a dual basis with respect to the two-point functions:

$$
\left\langle x_{i} x^{j}\right\rangle=\delta_{i}^{j}
$$

The associativity of the multiplication (2.5) follows from the duality 3 of the four-point correlation functions. For a more profound review of the physics of the $\sigma$-models on the Kähler manifolds we refer our reader to [22] and references therein.

2 We impose a mass shell condition $F_{\alpha}^{i}=0$ on the auxiliary field.

3 This duality has nothing to do with (2.6) 


\subsection{Vertical quantum cohomology.}

In this paper we discuss the functoriality properties of what we call the vertical quantum cohomology. This object will be rigorously defined in the subsequent section; here we introduce it in one particular setup using the language of the topological $\sigma$-model. Loosely speaking, the vertical quantum cohomology appear in a situation when there is an algebraic bundle or more generally, a family of deformations with a "quantum" fiber and a "classical" base. The classical limit (large radius limit for the fiber) of the vertical quantum cohomology ring is just the ordinary de Rham cohomology ring of the total space.

Let $X$ be a simply connected compact Kähler manifold with a structure of a locally trivial algebraic bundle over a simply connected compact Kähler manifolds $B$ (the base) with fiber $F$ and with a projection of the total space $\pi: X \rightarrow B$. In this setup, according to Deligne [24], [25], the Leray spectral sequence for the bundle $\pi: X \rightarrow B$ degenerates in the second term. This means that $H^{*}(B)$ is a subring in the cohomology ring $H^{*}(X)$ of the total space and that as a $H^{*}(B)$-algebra $H^{*}(X)$ is generated by some elements $x_{1}, \ldots, x_{N} \in H^{*}(X)$ such that when restricted to a fiber they generate the whole ring $H^{*}(F)$.

We assume also that the first Chern classes $c_{1}(F)$ and $c_{1}(X)$ are non-negative. The result of multiplication of two elements in the quantum cohomology ring $Q H^{*}(X)$ is represented by a power series in quantum deformation parameters $q_{1}, \ldots, q_{n+m}$. From the point of view of the topological $\sigma$-model a parameter $q_{i}$, corresponding to a certain homological class $[\Sigma]_{i}$ of algebraic curves in $X$, is an exponential function

$$
q_{i}=\exp \left(-\int_{\Sigma} k\right)
$$

of the minus area of the curve in that class. A semi-group $\operatorname{ting} \mathbf{C}\left[H_{11}(X)\right]$ is canonically imbedded into the ring of power series generated by the deformation parameters $\mathbf{K}=$ $\mathbf{C}\left[\left[q_{1}, \ldots, q_{n+m}\right]\right]$. It is convenient to think of the ring $Q H^{*}(X)$ as a $\mathbf{K}$-algebra.

Now we wish to utilize a structure of $X$ as a bundle over $B$ in a simple way: we take a "partial classical limit" of the ring $Q H^{*}(X)$ "along the base". To define this limit let us take a form $k_{h} \in H^{11}(B) \subset H^{11}(X)$ in the interior of the Kähler cone of $X$ and let

4 A semi-group $H_{11}(X)$ is generated by the homological classes of algebraic curves in $X$ taken with nonnegative integer coefficients. To a group operation in $H_{11}(X)$ there corresponds a multiplication in the semi-group ring. 
us consider a one-parametric family of the Kähler classes represented by $k(\lambda)=k+\lambda k_{h}$, $\lambda \geq 0$. Then the "partial classical limit" corresponds to

$$
\lambda \longrightarrow \infty
$$

A semi-group $H_{11}(X)$ has a sub-semi-group $\mathcal{V}$ generated 5 by the homological classes of curves taking zero values on $k_{h}$. These are vertical curves: the projection $\pi: X \rightarrow B$ send them to points on the base. There is an ideal $I$ in the semi-group ring of $H_{11}(X)$ generated by the set-theoretical complement to $\mathcal{V}$ in $H_{11}(X)$ :

$$
I=\mathbf{C}\left[H_{11}(X) \backslash \mathcal{V}\right]
$$

It consists exactly of those polynomials in $q_{1}, \ldots, q_{n+m}$ that tend to zero in the limit (2.8)if we substitute the expressions (2.7) for $\left\{q_{i}\right\}$.

We can define a quotient ring

$$
\tilde{\mathbf{K}}=\mathbf{C}\left[\left[q_{1}, \ldots, q_{n+m}\right]\right] / I=\mathbf{C}\left[\left[q_{n+1}, \ldots, q_{n+m}\right]\right]
$$

which is related to the semi-group ring $\mathbf{C}\left[H_{11}(F)\right]$ as $\mathbf{K}$ is related to $\mathbf{C}\left[H_{11}(X)\right]$. The quotient

$$
Q H_{V}^{*}(X, B, F)=Q H^{*}(X) / I
$$

is a $\tilde{\mathbf{K}}$-algebra. This is what we mean by the limit $(2.8)$ of the ring $Q H^{*}(X)$. We call a ring $Q H_{V}^{*}(X, B, F)$ the vertical quantum cohomology ring of the bundle $\pi: X \rightarrow B$.

To investigate the limit (2.8) in more detail we can consider a topological (type A) model with a world sheet $\Sigma$ and a target space $X$. The $m$-point correlation functions there are given by the path integral

$$
\left\langle\mathcal{O}_{\omega_{1}} \cdots \mathcal{O}_{\omega_{m}}\right\rangle=\int \mathcal{D} X^{i} \mathcal{D} \chi^{i} \mathcal{D} \rho_{\alpha}^{i} \mathcal{D} F_{\alpha}^{i} \mathcal{O}_{\omega_{1}} \cdots \mathcal{O}_{\omega_{m}} e^{A[X, \chi, \rho, F]}
$$

where the operators $\mathcal{O}_{\omega_{i}}$ are given by (2.4). The action functional $A$ is a sum of two pieces:

$$
A=\int \phi^{*} k+t\left\{Q, \int \frac{1}{2} g^{\alpha \beta} G_{i j} \rho_{\alpha}^{i}\left(\partial_{\alpha} X^{j}-\frac{1}{2} F_{\beta}^{j}\right)\right\}
$$

The first term depends only on the homological type of the map $\phi: \Sigma \rightarrow X$ and on the cohomology class of the Kähler form $k$ of $X$. The second term does not contribute to

5 In fact $\mathcal{V}=H_{11}(F)$. 
the correlation functions, since it is BRST-trivial. Therefore, it suffices to compute the integral (2.12) in the limit $t \longrightarrow \infty$. In this limit the measure $\left[\mathcal{D} X^{i} \mathcal{D} \chi^{i} \mathcal{D} \rho_{\alpha}^{i} \mathcal{D} F_{\alpha}^{i}\right] e^{A[X, \chi, \rho, F]}$ is localized to the holomorphic maps $6: \Sigma \rightarrow X$.

The correlation function (2.12) depends on $\lambda$ via the exponential term $\exp \left(-\lambda \int_{\Sigma} \phi^{*} k_{h}\right)$. In the limit $\lambda \longrightarrow \infty$ the contributions to (2.12) from all except the vertical maps $\phi_{V}: \Sigma \rightarrow X$ are suppressed by the factor $e^{-\lambda}$. Here the vertical maps are defined by the homological property to annihilate (any) horizontal Kähler form. (This is equivalent to a condition that the composition $\pi \circ \phi_{V}: \Sigma \rightarrow B$ maps the whole curve $\Sigma$ to a point: $\left.\operatorname{Im}\left(\pi \circ \phi_{V}\right)=\{\cdot\}\right)$.

We see that in the limit (2.8) the integration in (2.12) reduces to the integration over zero modes of the "horizontal" fields plus integration over the vertical holomorphic maps:

$$
\begin{aligned}
& \left\langle\mathcal{O}_{\omega(1)} \cdots \mathcal{O}_{\omega(m)}\right\rangle= \\
& \int_{[B]} \int \mathcal{D} X_{x}^{I} \mathcal{D} \chi^{I} \mathcal{D} \rho_{\alpha}^{I} e^{A[X, \chi, \rho]} \omega_{i_{1} \cdots i_{k_{1}} I_{1} \cdots I_{l_{1}}}^{(1)}\left(x, X_{x}(z)\right) d x^{i_{1}} \ldots d x^{i_{k_{1}}} \chi^{I_{1}}(z, \bar{z}) \ldots \chi^{I_{l_{1}}}(z, \bar{z}) \\
& \cdots \omega_{j_{1} \cdots j_{k_{m}} J_{1} \cdots J_{l_{m}}}^{(m)}\left(x, X_{x}(z)\right) d x^{j_{1}} \ldots d x^{j_{k_{m}}} \chi^{J_{1}}(z, \bar{z}) \ldots \chi^{J_{l_{m}}}(z, \bar{z})
\end{aligned}
$$

In this formula the coordinates along the base are denoted by $x^{i}$ and the coordinates along the fiber by $X_{x}^{I}$. The latter are only defined over each single point of the base which we stressed by putting a subscript $x$.

The space of the vertical holomorphic maps is fibered over $B$ with a space of holomorphic maps $\mathcal{M}_{F}=\{\Sigma \rightarrow F\}$ as a fiber. The second integration in (2.14)is performed with $x$ fixed; it goes over the fiber $\mathcal{M}_{\pi^{-1}(x)}$. Thus its result is a correlation function in the topological $\sigma$-model with the fiber $F$ as a target space.

The integral $\int_{[B]}$ is an ordinary integral over the base of the differential form on $B$ which we call the vertical correlation function and denote by $\left\langle\mathcal{O}_{\omega^{(1)}} \cdots \mathcal{O}_{\omega^{(m)}}\right\rangle_{(v)}$, so finally

$$
\left\langle\mathcal{O}_{\omega^{(1)}} \cdots \mathcal{O}_{\omega^{(m)}}\right\rangle=\int_{[B]}\left\langle\mathcal{O}_{\omega^{(1)}} \cdots \mathcal{O}_{\omega^{(m)}}\right\rangle_{(v)}
$$

The $m$-point vertical correlation functions are defined for all $m$-tuples $\mathcal{O}_{\omega^{(1)}} \cdots \mathcal{O}_{\omega^{(m)}}$ of operators in the limit $\lambda \longrightarrow \infty$ of the topological $\sigma$-model by the second integral in (2.14). From the definition it is clear that they satisfy

$$
\left\langle\cdots \mathcal{O}_{\omega}\right\rangle_{(v)}=\langle\cdots\rangle_{(v)} \wedge \omega
$$

6 Note then that the first term in (2.13) computes the area of the world sheet in the pullback metrics $\phi^{*} G_{i j}$. 
for any $\omega \in H^{*}(B) \subset H^{*}(X)$. This means that the operator algebra of the topological $\sigma$-model in the limit (2.8) has a subalgebra isomorphic to the classical cohomology ring of the base $H^{*}(B)$. It shows that the vertical quantum cohomology ring $Q H_{V}^{*}(X, B, F)$ is actually an $H^{*}(B)$-algebra.

What makes the ring $Q H_{V}^{*}(X, B, F)$ a rather interesting object is that it behaves nicely with respect to the change of the base operation. Let $X^{\prime}$ be a fiber product of $B^{\prime}$ and $F$ induced from $X$ by a morphism $i: B^{\prime} \rightarrow B$ of the basest. Then there are pullback morphisms $i^{*}: H^{*}(B) \rightarrow H^{*}\left(B^{\prime}\right)$ and $j^{*}: H^{*}(X) \rightarrow H^{*}\left(X^{\prime}\right)$. By definition, $Q H_{V}^{*}(X, B, F)$ is an algebra over $H^{*}(B)$ and $Q H_{V}^{*}\left(X^{\prime}, B^{\prime}, F\right)$ is an algebra over $H^{*}\left(B^{\prime}\right)$. A pullback $j^{*}$ induces a pullback morphism $j_{q}^{*}: Q H_{V}^{*}(X, B, F) \rightarrow Q H_{V}^{*}\left(X^{\prime}, B^{\prime}, F\right)$ of vector spaces. We claim that in fact $j_{q}^{*}$ is a morphism of rings.

\section{Base change for the vertical quantum cohomology.}

As an $H^{*}\left(B^{\prime}\right)$-algebra, $j_{q}^{*}\left(Q H_{V}^{*}(X, B, F)\right) \otimes_{H^{*}(B)} H^{*}\left(B^{\prime}\right)$ is a subalgebra in $Q H_{V}^{*}\left(X^{\prime}, B^{\prime}, F\right)$.

Example. The following (trivial) example demonstrates one standard application of the base change property. Consider an imbedding $\{p t\} \rightarrow B$ inducing a trivial bundle $(F,\{p t\}, F)$ with vertical quantum cohomology $Q H_{V}^{*}(F,\{p t\}, F)=Q H^{*}(F)$ given by the usual quantum cohomology of the fiber $F$. The base change property gives $Q H_{V}^{*}(F,\{p t\}, F)=Q H_{V}^{*}(X, B, F) / L$ where $L$ is an ideal in $H^{*}(B) \subset Q H_{V}^{*}(X, B, F)$ generated by $\oplus_{i>0} H^{i}(B)$. Thus we have the identity

$$
Q H^{*}(F)=Q H_{V}^{*}(X, B, F) / L
$$

which relates a partial classical limit of quantum cohomology of the total space $X$ and the quantum cohomology of the fiber $F$.

Remark. Actually for the definition of the vertical quantum cohomology it is not necessary that the base $B$ be Kähler or compact. For any algebraic $B$, it is possible to define this ring and it still has a structure of $H^{*}(B)$-algebra. For a physicist, the most natural example would be a family of Calabi-Yau manifolds considered over some open set in the moduli space of their complex structures. But in this paper we discuss another example based on geometry of the algebraic vector bundles.

7 In other words, $X^{\prime}$ is defined as $X \times_{B} B^{\prime}$. 
2.3. Equivariant quantum cohomology and quantum cohomology of the partial flag manifolds.

An interesting application of the base change property appears in a situation when $X$ is a bundle of partial flag manifolds $F_{n_{1} \cdots n_{k}}(E)$ associated with a complex holomorphic rank $n$ vector bundle $E$ on $B$; the partial flag manifold is defined as the quotient $F_{n_{1} \cdots n_{k}}=U(n) /\left(\otimes_{i=1}^{k} U\left(n_{i}\right)\right)$. In particular, this may be a projectivization $\mathbf{P}(E)$. There is a classical theory of cohomology of such bundles [26] which effectively reduces to the following statement. Consider a map $i: B \rightarrow B U(n)$ of the base $B$ of $E$ to the classifying space $B U(n)$; then the ring $H^{*}\left(F_{n_{1} \cdots n_{k}}(E)\right)$ as a $H^{*}(B)$-algebra is obtained as a pullback $i^{*}$ of the cohomology ring of the universal $F_{n_{1} \cdots n_{k}}$ flag bundle on $B U(n)$. This latter ring is what is called the $U(n)$-equivariant cohomology $H_{U(n)}^{*}\left(F_{n_{1} \cdots n_{k}}\right)$ of the flag variety. The ring $H_{U(n)}^{*}\left(F_{n_{1} \cdots n_{k}}\right)$ is an algebra over $H^{*}(B U(n))=\mathbf{C}\left[c_{1}, \ldots, c_{n}\right]$ which is a graded polynomial algebra generated by the Chern classes $c_{1}, \ldots, c_{n}$ of the vector bundle, associated to the universal bundle $E U(n) \rightarrow B U(n)$. The pullback morphism $i^{*}: H^{*}(B U(n)) \rightarrow H^{*}(B)$ maps the generators $c_{i} \longrightarrow c_{i}(E)$ to the Chern classes of the bundle $E$.

For example, for the projective space $\mathbf{P}^{n-1}$ the equivariant cohomology ring is given by

$$
H_{U(n)}^{*}\left(\mathbf{P}^{n-1}\right)=H^{*}(B U(n))[x] /\left(x^{n}+c_{1} x^{n-1}+\cdots+c_{n}\right)
$$

Taking the pullback $i^{*}$, we get the cohomology ring of the projectivization $\mathbf{P}(E)$ :

$$
H^{*}(\mathbf{P}(E))=H^{*}(B)[x] /\left(x^{n}+c_{1}(E) x^{n-1}+\cdots+c_{n}(E)\right)
$$

where $c_{i}(E)$ is the i-th Chern class of $E$.

The base change property of the vertical quantum cohomology ensures the same holds true in quantum case, if one uses quantum equivariant cohomology instead of equivariant ones. It is the equivariant cohomology of the complete flag manifolds $F_{n}$ that was computed in [1]. We can formulate the definition of [1] in terms of vertical quantum cohomology as follows: for any $G$-manifold $F$ the quantum equivariant cohomology ring is defined as

$$
Q H_{G}^{*}(F)=Q H_{V}^{*}\left(F \times_{G} E G, B G, F\right)
$$

The ring $Q H_{U(n)}^{*}\left(F_{n_{1} \cdots n_{k}}\right)$ will be computed in section 5. To describe it, let us introduce the generators $x_{1}^{(1)}, \ldots, x_{n_{1}}^{(1)}, \ldots, x_{1}^{(k)}, \ldots, x_{n_{k}}^{(k)}$ of degrees deg $x_{i}^{(j)}=2 i$ and the quantum deformation parameters $q_{1}, \ldots, q_{k-1}$ of degrees deg $q_{i}=n_{i}+n_{i+1}$. The ring 
$Q H_{U(n)}^{*}\left(F_{n_{1} \cdots n_{k}}\right)$ is a quotient by a homogenous ideal $Q I$ of the graded polynomial algebra generated over $H^{*}(B U(n))$ by $\left\{x_{i}^{(j)}, q_{j}\right\}$ :

$$
Q H_{U(n)}^{*}\left(F_{n_{1} \cdots n_{k}}\right)=H^{*}(B U(n))[x, q] / Q I
$$

The ideal $Q I$ is generated by the coefficients of the polynomial

$$
\operatorname{det}[\lambda \mathbf{1}+A]-\lambda^{n}-c_{1} \lambda^{n-1}-\ldots-c_{n}
$$

where the $n \times n$ matrix $A$ is defined as

$$
\left(\begin{array}{cccccccccccc}
x_{1}^{(1)} & \ldots & x_{n_{1}}^{(1)} & 0 & \ldots & -(-1)^{n_{2}} q_{1} & \ldots & 0 & \ldots & \ldots & 0 & 0 \\
-1 & \ldots & 0 & 0 & \ldots & 0 & \ldots & 0 & \ldots & \ldots & 0 & 0 \\
\vdots & \ddots & \vdots & \vdots & \ddots & \vdots & \ddots & \vdots & \ddots & \ddots & \vdots & \vdots \\
0 & \ldots & -1 & x_{1}^{(2)} & \ldots & x_{n_{2}}^{(2)} & \ldots & -(-1)^{n_{3}} q_{2} & \ldots & \ldots & 0 & 0 \\
\vdots & \ddots & \vdots & \vdots & \ddots & \vdots & \ddots & & \ddots & \ddots & \vdots & \vdots \\
\vdots & \ddots & \vdots & \vdots & \ddots & \vdots & \ddots & & \ddots & \ddots & \vdots & \vdots \\
\vdots & \ddots & \vdots & \vdots & \ddots & \vdots & \ddots & \ddots & & \ddots & \vdots & \vdots \\
\vdots & \ddots & \vdots & \vdots & \ddots & \vdots & \ddots & \ddots & & \ddots & \vdots & \vdots \\
0 & \ldots & 0 & 0 & \ldots & 0 & \ldots & \ldots & x_{1}^{(k)} & \ldots & x_{n_{k}-1}^{(k)} & x_{n_{k}}^{(k)} \\
0 & \ldots & 0 & 0 & \ldots & 0 & \ldots & \ldots & -1 & \ldots & 0 & 0 \\
\vdots & \ddots & \vdots & \vdots & \ddots & \vdots & \ddots & \ddots & \vdots & \ddots & \vdots & \vdots \\
0 & \ldots & 0 & 0 & \ldots & 0 & \ldots & \ldots & 0 & \ldots & -1 & 0
\end{array}\right)
$$

The entries of $A$ are graded according to the number of the diagonal they belong to: those on the diagonal just below the main diagonal have degree 0 , those on the main diagonal has degree 2 etc. All the entries on the diagonal just below the main one equal $(-1)$ and yet below everything equal 0 . There are non-zero entries on the main diagonal and above in the 1-st, $n_{1}$-th, $n_{1}+n_{2}$-th, $\ldots, n_{1}+\cdots+n_{k-1}$-th rows:

$$
A_{\left(n_{1}+\cdots+n_{i-1}, n_{1}+\cdots+n_{i-1}+j\right)}= \begin{cases}-1, & \text { if } j=-1 \\ x_{j}^{(i)}, & \text { if } 0 \leq j \leq n_{i} \\ -(-1)^{n_{i+1}} q_{i}, & \text { if } j=n_{i}+n_{i+1} \\ 0, & \text { otherwise }\end{cases}
$$

Taking the pullback $i^{*}$, we obtain the vertical quantum cohomology of the bundle $F_{n_{1} \cdots n_{k}}(E)$ as an algebra generated over $H^{*}(B)$ by the same generators $x_{1}^{(1)}, \ldots, x_{n_{k}}^{(k)}$ and 
quantum deformation parameters $q_{1}, \ldots, q_{k-1}$ as above with relations given by the coefficients of polynomial

$$
\operatorname{det}[\lambda \mathbf{1}+A]-\lambda^{n}-c_{1}(E) \lambda^{n-1}-\ldots-c_{n}(E)
$$

Setting in $(2.24) c_{1}(E)=0, \ldots, c_{n}(E)=0$ (cf. (2.17)), one gets the relations in the quantum cohomology ring $Q H^{*}\left(F_{n_{1} \cdots n_{k}}\right)$ of the partial flag manifold $F_{n_{1} \cdots n_{k}}$.

Remark It should be noted that the situation with the $\operatorname{ring} Q H^{*}\left(F_{n_{1} \cdots n_{k}}(E)\right)$ is much less clear. We discuss this in Appendix A.

\section{A math introduction to vertical quantum cohomology.}

\subsection{Quantum cohomology.}

Let $X$ be a smooth complex projective variety, $c$ the first Chern class of the tangent bundle of $X$. Now and for all we assume that $c$ is non-negative. Let us choose some basis $\omega_{1}, \omega_{2}, \ldots, \omega_{k}$ in $H^{2}(X, \mathbf{Z}) \cap H^{1,1}(X, \mathbf{C})$ such that the homology class of a complex curve $S$ is given by a string $d=\left(d_{1}, \ldots, d_{k}\right)$ of its coordinates with respect to $\omega_{1}, \omega_{2}, \ldots, \omega_{k}$, with $d_{1}, \ldots, d_{k} \geq 0$. Let $L$ be a sublattice in $H_{2}(X, \mathbf{Z}) \cap H_{2}(X, \mathbf{C})$ generated by the homology classes of complex curves $\left(\mathbf{C P}^{1}\right)$. We use the notation $q^{d}$ for the elements of the group ring of the lattice $L$. Since we have already chosen some basis in $H^{2}(X, \mathbf{Z}) \cap H^{1,1}(X, \mathbf{C})$, we can identify the element $q^{d}$ with the monomial $q_{1}^{d_{1}} \ldots q_{k}^{d_{k}}$. We denote by $\mathbf{K}$ the ring $\mathbf{C}\left[\left[q_{1}, \ldots, q_{k}\right]\right]$ ( but see Appendix C ).

Let $d$ be an element of $L$. We denote by $\mathcal{M}_{d}$ the moduli space of degree $d$ algebraic maps $\mathbf{C P}^{1} \rightarrow X$ with fixed three points $(0,1, \infty)$. In our case $\mathcal{M}_{d}$ is a well-defined quasiprojective algebraic variety ( see Appendix D ). The Riemann-Roch formula tells us that $\operatorname{dim} \mathcal{M}_{d} \geq c(d)+\operatorname{dim} X$. We assume in this paper that for $X$ the equality holds, i.e. $\operatorname{dim} \mathcal{M}_{d}=c(d)+\operatorname{dim} X$.

Let $p_{1}, p_{2}, \ldots, p_{s}$ be closed differential forms on $X$ of degree $r_{1}, \ldots, r_{s}$ respectively. We want to define the correlation function $\left\langle p_{1}\left|p_{2}\right| \ldots \mid p_{s}\right\rangle$ now. For that purpose let us choose $s$ (different) generic points $x_{1}, \ldots, x_{s}$ on $\mathbf{C P}^{1}$. For each $d \in L$, we have a map $\psi: \mathcal{M}_{d} \rightarrow X^{s}$. It is given by

$$
\psi: \phi \mapsto\left(\phi\left(x_{1}\right), \phi\left(x_{2}\right), \ldots, \phi\left(x_{s}\right)\right) \in X^{s} \quad \text { where } \quad \phi \in \mathcal{M}_{d}
$$

Note that this is an algebraic map. We have $s$ canonical projections $\pi_{i}(i=1, \ldots, s)$ from $X^{s}$ to $X$. So we have the differential form $P=\pi_{1}{ }^{*} p_{1} \wedge \pi_{2}{ }^{*} p_{2} \wedge \ldots \wedge \pi_{s}{ }^{*} p_{s}$ on $X^{s}$. 
Definition. The correlation function $\left\langle p_{1}\left|p_{2}\right| \ldots \mid p_{s}\right\rangle$ is defined by

$$
\left\langle p_{1}\left|p_{2}\right| \ldots \mid p_{s}\right\rangle=\sum_{d} q^{d} \int_{\mathcal{X}_{d}} \psi^{*} P
$$

First of all, we must show that this definition is correct. In order to do that we must show that the integral $\int_{\mathcal{M}_{d}} \psi^{*} P$ is well-defined. But this is easy to do. Indeed, we are integrating a differential form of the highest degree, so we can integrate it over any affine subvariety $U$ of $\mathcal{M}_{d}$ such that $\operatorname{dim}\left(\mathcal{M}_{d}-U\right)<\operatorname{dim} \mathcal{M}_{d}$. Second, let us distinguish two cases:

1) the dimension of the closure of $\psi\left(\mathcal{M}_{d}\right)$ is less then the dimension of $\mathcal{M}_{d}$

2) the dimension of the closure of $\psi\left(\mathcal{M}_{d}\right)$ is the same as the dimension of $\mathcal{M}_{d}$.

In the first case it is quite obvious that the integral is equal to 0 . In the second case we can find such affine subvariety $U$ of $\mathcal{M}_{d}$ satisfying the above condition that the restriction of $\psi$ to $U$ gives a covering of the image $\psi(U)$. So we have

$$
\int_{\mathcal{M}_{d}} \psi^{*} P=\int_{U} \psi^{*} P=\operatorname{deg}(\psi) \int_{\psi(U)} P .
$$

The last integral is well-defined (since $\psi(U)$ is a constructible set).

Second, we must show that the definition (3.1)does not depend on the choice 8 of $x_{1}, \ldots, x_{s}$ on $\mathbf{C P}^{1}$. A reader may find the proof in [27].

\section{Properties of correlation functions:}

1. They are multi-linear and skew-symmetric.

2. $\left\langle p_{1}\left|p_{2}\right| \ldots\left|p_{s}\right| 1\right\rangle=\left\langle p_{1}\left|p_{2}\right| \ldots \mid p_{s}\right\rangle$ (where 1 is considered as a zero degree differential form).

3. $\left.\left\langle p_{1}\left|p_{2}\right| \ldots \mid p_{s}\right\rangle\right|_{q=0}=\left(p_{1}, \ldots, p_{s}\right)$ where $\left(p_{1}, \ldots, p_{s}\right)=\int_{X} p_{1} \wedge p_{2} \wedge \ldots \wedge p_{s}$.

4. $\langle p\rangle=(p)=\int_{X} p$.

5. $\left\langle p_{1} \mid p_{2}\right\rangle=\left(p_{1}, p_{2}\right)$ is the usual Poincaré pairing.

All properties follow obviously from the definition except the last one. Let us explain why the last property is true. We choose two points $x_{1}$ and $x_{2}$ of $\mathbf{C P}^{1}$. The group $\mathbf{C}^{*}$ is the group of automorphisms of $\mathbf{C P}^{1}$ with two fixed points $x_{1}$ and $x_{2}$. It acts on the variety $\mathcal{M}_{d}$, and the map $\psi$ from the definition is constant on the $\mathbf{C}^{*}$-orbits. The unique

8 We can integrate over the set of all possible choices of points (i.e. over $\mathbf{C P}^{s}$ ), so the definition does not depend on the choice of points 
non-empty variety $\mathcal{M}_{d}$ on which $\mathbf{C}^{*}$ acts trivially is $\mathcal{M}_{0}=X$. On the other moduli spaces the action is "free almost everywhere". So the dimension of the closure of the image of $\psi$ is less then that of $\mathcal{M}_{d}$ if $d \neq 0$. So we get the ordinary Poincaré pairing.

Now we define quantum cohomology. The Poincaré pairing gives us an isomorphism of $H^{*}(X, \mathbf{C}) \otimes \mathbf{K}$ with its dual as $\mathbf{K}$-modules. So the triple pairing gives a product:

$$
\left(H^{*}(X, \mathbf{C}) \otimes \mathbf{K}\right) \bigotimes_{\mathbf{K}}\left(H^{*}(X, \mathbf{C}) \otimes \mathbf{K}\right) \rightarrow H^{*}(X, \mathbf{C}) \otimes \mathbf{K}
$$

It is obvious that the product (3.2) is skew-commutative. We are not going to prove that this product is associative (as it is highly non-trivial. See [27]), but we assume that it is true. The product (3.2) gives us what we call quantum cohomology ring $Q H^{*}(X, \mathbf{K})$ of $X$. As $\mathbf{K}$-module, the quantum cohomology is isomorphic to $H^{*}(X, \mathbf{C}) \otimes \mathbf{K}$, but the products in these two rings are different.

\section{Properties 9 :}

1) the product (3.2) is a $q$-deformation of the classical product

2) the product (3.2) respects the usual grading in the cohomology $H^{*}(X, \mathbf{C}) \otimes \mathbf{K}$ (remember that $\mathbf{K}$ is a graded ring).

\subsection{Vertical quantum cohomology.}

Let us suppose 10 that we have a locally trivial algebraic fiber bundle $X$ with a base $B$ and a fiber $F$. We assume that all $X, B$ and $F$ satisfy all the conditions imposed on the varieties for which we have defined the quantum cohomology. Deligne's theorem (see [24]) tells that the corresponding Leray spectral sequence degenerates in $E_{2}$. So the map from $H^{2}(X, \mathbf{C})$ to $H^{2}(F, \mathbf{C})$ is surjective. Denote the projection from $X$ to $B$ by $\pi$ and an inclusion of $F$ into $X$ by $i$.

Let $\tilde{\omega}_{1}, \ldots, \tilde{\omega}_{k}$ be our chosen basis in $H^{2}(F, \mathbf{Z}) \cap H^{1,1}(F, \mathbf{C})$ for $F, \tilde{\omega}_{k+1}, \ldots, \tilde{\omega}_{l}$ basis for $B$. We can find a basis $\omega_{1}, \ldots, \omega_{k}, \omega_{k+1}, \ldots, \omega_{l}$ for $X$ such that $\omega_{i}=\pi^{*}\left(\tilde{\omega}_{i}\right)$ for $i>k$ and $\tilde{\omega}_{i}=i^{*}\left(\omega_{i}\right)$ for $i \leq k+1$. Certainly the choice is not unique but nothing essential would depend on it. We want to deform the usual multiplication using only the maps from $\mathbf{C P}^{1}$ to $X$ which project onto points in $B$. We call such maps vertical. The homology class of

\footnotetext{
9 see [1]

10 In fact, vertical quantum cohomology can be defined in more general situation. But we need it only in this case and it is easier to describe it (really the ring $\mathbf{K}$ ) in this particular situation.
} 
such a map can be parametrized by a string $d=\left(d_{1}, \ldots, d_{k}\right)$, the evaluation of $\omega_{1}, \ldots, \omega_{k}$ on the image. So the deformation will be defined over the $\operatorname{ring} \tilde{\mathbf{K}}=\mathbf{C}\left[\left[q_{1}, \ldots, q_{k}\right]\right]$.

We denote the moduli space of the vertical maps of degree $d$ by $\mathcal{M}_{d}^{(v)}$. One can see that $\mathcal{M}_{d}^{(v)}$ projects onto $B$ and the fiber is $\mathcal{M}(F)_{d}$. Let us denote this projection by $\tau$.

Now we can define vertical correlation functions in the same way we did before to define (3.1). We choose $s$ generic points $x_{1}, \ldots, x_{s}$ on $\mathbf{C P}^{1}$. This gives us a map from $\mathcal{M}_{d}^{(v)}$ to $X^{s}$. Denote it by $\mu$. Let $p_{1}, p_{2}, \ldots, p_{s}$ be closed differential forms on $X$ of degree $r_{1}, \ldots, r_{s}$ respectively. Denote by $P$ the differential form $\pi_{1}{ }^{*} p_{1} \wedge \pi_{2}{ }^{*} p_{2} \wedge \ldots \wedge \pi_{s}{ }^{*} p_{s}$.

Definition. The vertical correlation function $\left\langle p_{1}\left|p_{2}\right| \ldots \mid p_{s}\right\rangle_{(v)}$ is given by

$$
\left\langle p_{1}\left|p_{2}\right| \ldots \mid p_{s}\right\rangle_{(v)}=\sum_{d} q^{d} \tau_{!}\left(\mu^{*} P\right)
$$

where $\tau_{!}$means the integration over fibers. It takes values in the cohomology of $B$, i.e. in $H^{*}(B, \tilde{\mathbf{K}})$.

In the same way as for the definition (3.1) of the ordinary correlation functions, one can prove correctness of this definition.

\section{Properties of the vertical correlation functions:}

1. They are multi-linear and skew-symmetric.

2. $\left\langle p_{1}\left|p_{2}\right| \ldots\left|p_{s}\right| 1\right\rangle_{(v)}=\left\langle p_{1}\left|p_{2}\right| \ldots \mid p_{s}\right\rangle_{(v)}$ (where 1 is considered as a zero degree differential form).

3. $\left.\left\langle p_{1}\left|p_{2}\right| \ldots \mid p_{s}\right\rangle_{(v)}\right|_{q=0}=\pi_{!}\left(p_{1} \wedge \ldots \wedge p_{s}\right)$.

4. $\langle p\rangle_{(v)}=\pi_{!}(p)$.

5. $\left\langle p_{1} \mid p_{2}\right\rangle_{(v)}=\pi_{!}\left(p_{1} \wedge p_{2}\right)$.

6. Let $p$ be any differential form on $B$. Then $\left\langle\pi^{*} p \wedge p_{1}\left|p_{2}\right| \ldots \mid p_{s}\right\rangle_{(v)}=p \wedge\left\langle p_{1}\left|p_{2}\right| \ldots \mid p_{s}\right\rangle_{(v)}$

All properties except the last one can be proved in the same way as in the ordinary case.

The last property is quite obvious and follows from the functoriality and the properties of the functors $\tau_{!}, \pi_{!}$and $\pi^{*}$.

We can define the vertical quantum cohomology now. The Poincaré pairing on $X$ gives us an isomorphism of $H^{*}(X, \mathbf{C}) \otimes \tilde{\mathbf{K}}$ with its dual as $\tilde{\mathbf{K}}$-modules. In order to define the product we must have a triple pairing with values in $\tilde{\mathbf{K}}$. We already have a triple pairing (3.3) with values in $H^{*}(B, \tilde{\mathbf{K}})$. So we can define the auxiliary triple pairing by means of integration of (3.3) over the fundamental cycle $B$ :

$$
\left\langle p_{1}\left|p_{2}\right| p_{3}\right\rangle_{\text {auxiliary }}=\int_{B}\left\langle p_{1}\left|p_{2}\right| p_{3}\right\rangle_{(v)} .
$$


This gives us a product. Certainly this product is skew-commutative. It is not obvious at all that this product is associative, but we will prove it below. Let us denote this product by $*$ and the algebra of vertical quantum cohomology by $Q H_{V}^{*}(X, \tilde{\mathbf{K}})$ (or sometimes by $Q H_{V}^{*}(X ; B, \tilde{\mathbf{K}})$, in order to avoid confusion).

First of all, let us notice the following property of the multiplication $*$ which shows that it is compatible with the vertical correlation functions (3.3):

$$
\left\langle p_{1}\left|p_{2}\right| p_{3}\right\rangle_{(v)}=\left\langle p_{1} * p_{2} \mid p_{3}\right\rangle_{(v)} .
$$

This follows directly from the definition.

The proof of the associativity is based on the relation between the quantum cohomology of $X$ and the vertical quantum cohomology. One can get the following formula from the definitions:

$$
\left.\left\langle p_{1}\left|p_{2}\right| \ldots \mid p_{s}\right\rangle\right|_{q_{k+1}=0, \ldots, q_{l}=0}=\left\langle p_{1}\left|p_{2}\right| \ldots \mid p_{s}\right\rangle_{(v)} .
$$

This shows that the vertical quantum product is equal to the usual quantum product on $X$ when we set $q_{k+1}=0, \ldots, q_{l}=0$. Since the usual quantum product is associative, this ends the proof of associativity. This relation between the vertical quantum cohomology and the usual quantum cohomology is a particular case of a certain property of the vertical quantum cohomology, the so-called induction, which we will discuss a bit later.

\subsection{Properties of the vertical quantum cohomology.}

\section{Product}

Suppose that we have two locally trivial algebraic fiber bundles $\left(X_{1}, B_{1}, F_{1}\right)$ and $\left(X_{2}, B_{2}, F_{2}\right)$ for which the vertical quantum cohomology is defined. The product $(X, B, F)=\left(X_{1} \times X_{2}, B_{1} \times B_{2}, F_{1} \times F_{2}\right)$ of these bundles is also a locally trivial algebraic fiber bundle. Then for the vertical quantum cohomology of the product 11 we have a formula

$$
Q H_{V}^{*}\left(X, \tilde{\mathbf{K}}_{1} \otimes_{\mathbf{C}} \tilde{\mathbf{K}}_{2}\right)=Q H_{V}^{*}\left(X_{1}, \tilde{\mathbf{K}}_{1}\right) \otimes_{\mathbf{C}} Q H_{V}^{*}\left(X_{2}, \tilde{\mathbf{K}}_{2}\right)
$$

This statement is trivial; it follows immediately from the fact that the moduli spaces of vertical maps of $\mathbf{C P}^{1}$ to $X$ are the products of the moduli spaces for $X_{1}$ and $X_{2}$ ( and that the Poincaré pairing on the cohomology of $X$ is the product of the Poincaré pairings on $X_{1}$ and $\left.X_{2}\right)$.

11 One can see that the vertical quantum cohomology of $\mathrm{X}$ is a module over $\tilde{\mathbf{K}}_{1} \otimes_{\mathbf{C}} \tilde{\mathbf{K}}_{2}$. 


\section{Restriction}

Consider a locally trivial algebraic bundle $(X, B, F)$ and an algebraic map from $\bar{B}$ to $B$. Take the Cartesian product of $X$ and $\bar{B}$ over $B$. Denote this product by $Y$ and the natural map from $Y$ to $X$ by $\nu$. Then $Y$ is a locally trivial algebraic fiber bundle over $\bar{B}$ with the fiber $F$. The cohomology $H^{*}(Y, \mathbf{C})$ is generated by the cohomology of $\bar{B}$ and the image of $H^{*}(X, \mathbf{C})$ ( this is true since the spectral sequence for $Y \rightarrow \bar{B}$ degenerates in $E_{2}$ (Deligne's theorem see [24, 225])). We have the part $\omega_{1}, \ldots, \omega_{k}$ of a basis in $H^{2}(X, \mathbf{Z}) \cap H^{1,1}(X, \mathbf{C})$. We can choose $\bar{\omega}_{1}=\nu^{*}\left(\omega_{1}\right), \ldots, \bar{\omega}_{k}=\nu^{*}\left(\omega_{k}\right)$ as the corresponding part of a basis in $H^{2}(Y, \mathbf{Z}) \cap H^{1,1}(Y, \mathbf{C})$. Then we get an isomorphism of the rings $\tilde{\overline{\mathbf{K}}}=\tilde{\mathbf{K}}$. Now we can say that we have a map:

$$
Q H_{V}^{*}(X, \tilde{\mathbf{K}}) \rightarrow Q H_{V}^{*}(Y, \tilde{\mathbf{K}}) .
$$

This map is just $\nu^{*}$ (since we have a natural isomorphism of $\tilde{\mathbf{K}}$-modules $Q H_{V}^{*}(X, \tilde{\mathbf{K}})$ and $\left.H^{*}(X, \tilde{\mathbf{K}})\right)$. Moreover, if we know the vertical cohomology of $X$, then we can compute the vertical cohomology of $Y$.

Certainly, we have a map between the vertical quantum cohomology as $\tilde{\mathbf{K}}$-modules. We must show that it respects the multiplication. But this is easy to see, since $\overline{\mathcal{M}}_{d}^{(v)}$ is equal to the Cartesian product of $\mathcal{M}_{d}^{(v)}$ and $\bar{B}$ over $B$. So we get:

$$
\left\langle\nu^{*} p_{1}\left|\nu^{*} p_{2}\right| \ldots \mid \nu^{*} p_{s}\right\rangle_{(v)}=\nu^{*}\left\langle p_{1}\left|p_{2}\right| \ldots \mid p_{s}\right\rangle_{(v)}
$$

Now we will use the fact that the vertical quantum product is uniquely defined by $\left\langle p_{1} * p_{2} \mid p_{3}\right\rangle_{(v)}=\left\langle p_{1}\left|p_{2}\right| p_{3}\right\rangle_{(v)}$ for any $p_{3}$. It is easy to check that $\left\langle\nu^{*}\left(p_{1} * p_{2}\right) \mid \bar{p}\right\rangle_{(v)}=$ $\left\langle\nu^{*} p_{1}\left|\nu^{*} p_{2}\right| \bar{p}\right\rangle_{(v)}$ for any $\bar{p}$ (here we use the fact that the usual cohomology is generated by the cohomology of $\bar{B}$ and the image of $\left.H^{*}(X, \tilde{\mathbf{K}})\right)$.

\section{Induction.}

Suppose that we have locally trivial algebraic bundles $\left(X, Y, F_{1}\right)$ and $\left(Y, B, F_{2}\right)$. Assume that the algebraic bundle $X \rightarrow B$ with the fiber $F$ is locally trivial. We choose a basis in $H^{2}(X, \mathbf{Z}) \cap H^{1,1}(X, \mathbf{C})$ in the following way: first, we choose a basis $\tilde{\omega}_{1}, \ldots, \tilde{\omega}_{m}$ for $F_{1}$; second, we choose a basis $\bar{\omega}_{1+m}, \ldots, \bar{\omega}_{k+m}, \bar{\omega}_{k+m+1}, \ldots, \bar{\omega}_{l+m}$ for $Y$ as before (when we were defining the vertical correlation functions ). If we denote now by $i$ an inclusion of $F_{1}$ into $X$ and by $\pi$ the projection from $X$ onto $Y$, then we can choose a basis $\omega_{1}, \ldots \omega_{l+m}$ in $H^{2}(X, \mathbf{Z}) \cap H^{1,1}(X, \mathbf{C})$ such that $i^{*}\left(\omega_{j}\right)=\tilde{\omega}_{j}$ for $1 \leq j \leq m$ and $\omega_{j}=\pi^{*}\left(\bar{\omega}_{j}\right)$ for $j>m$. Then denote by $\mathbf{K}$ the ring $\mathbf{C}\left[\left[q_{1}, \ldots, q_{k+m}\right]\right]$ and by $\tilde{\mathbf{K}}$ the $\operatorname{ring} \mathbf{C}\left[\left[q_{1}, \ldots, q_{m}\right]\right]$. 
In this situation the following relation between the $\operatorname{rings} Q H_{V}^{*}(X ; B, \mathbf{K})$ and $Q H_{V}^{*}(X ; Y, \tilde{\mathbf{K}})$ holds:

$$
\begin{aligned}
& Q H_{V}^{*}(X ; B, \mathbf{K}) /\left(\left(q_{m+1}, \ldots, q_{m+k}\right) Q H_{V}^{*}(X ; B, \mathbf{K})\right)=Q H_{V}^{*}(X ; Y, \tilde{\mathbf{K}}) \\
& \text { where }\left(q_{m+1}, \ldots, q_{m+k}\right) \text { is an ideal in } \mathbf{K} \text { generated by } q_{m+1}, \ldots, q_{m+k} \text {. }
\end{aligned}
$$

This fact follows from two simple observations. First ( we have already used this many times ), the product $*$ is defined uniquely by the correlation functions $\left\langle p_{1} * p_{2} \mid p_{3}\right\rangle_{(v)}=$ $\left\langle p_{1}\left|p_{2}\right| p_{3}\right\rangle_{(v)}$. Second, the moduli space $\mathcal{M}(X, B)_{\left(d_{1}, \ldots, d_{m}, 0, \ldots, 0\right)}^{(v)}$ coincides with the moduli space $\mathcal{M}(X, Y)_{\left(d_{1}, \ldots, d_{m}\right)}^{(v)}$. So we see that

$$
\left.\left\langle p_{1}\left|p_{2}\right| \ldots \mid p_{s}\right\rangle_{(v)}(X, B)\right|_{q_{m+1}=0, \ldots, q_{m+k}=0}=\left\langle p_{1}\left|p_{2}\right| \ldots \mid p_{s}\right\rangle_{(v)}(X, Y)
$$

This complets the sketch of the proof of induction.

Remark. If we look at the definition (3.3)of the vertical correlation functions, we will see that one can define them in the following situation. Let $G$ be a group acting on an algebraic variety $F, P$ a principal $G$ bundle over $B$ (we are not assuming here that $P$ is algebraic), and $P \times{ }_{G} F$ our fiber bundle. Then we can say that the moduli space of the vertical maps of degree $d$ is $P \times{ }_{G} \mathcal{M}(F)_{d}$. ( To make this statement precise we need to show that all the integrals converge; nevertheless it is useful to have this picture in mind. ) We will define equivariant quantum cohomology in the next section. One can think about them as a vertical quantum cohomology where $P$ is $E G$ and $B$ is $B G$ of the Lie group $G$. This picture and the properties of the vertical quantum cohomology explain the properties of the equivariant quantum cohomology.

\section{Equivariant quantum cohomology.}

Equivariant quantum cohomology were defined first in the paper [1]. Our approach is based on another, certainly equivalent, definition.

We use the setup of the previous chapter; $X$ is our algebraic variety. Suppose that we have an algebraic group $G$ acting on $F$. Denote by $g$ the Lie algebra of this group. As before, we denote by $\mathcal{M}_{d}$ the moduli space of degree $d$ maps from $\mathbf{C P}^{1}$ to $F$. Then the group $G$ acts on $\mathcal{M}_{d}$. 
Recall that the usual equivariant cohomology is defined as the cohomology of the following complex:

$$
C^{k}=\left(\oplus_{i+2 j=k} S^{j}(g)^{*} \otimes \Omega^{i}(F)\right)^{G}
$$

with the differential:

$$
\left(d_{g}(\alpha)\right)(h)=d(\alpha(h))-i(h)(\alpha(h)) \quad h \in g .
$$

The integration over the fundamental cycle of $F$ gives a map $\int$ from the equivariant cohomology of $X$ to the equivariant cohomology of the point $\left(H_{G}^{*}(p t ., \mathbf{C})\right)$. This map sends $C^{k}$ to $\left(S^{k-\operatorname{dim} F}(g)^{*}\right)^{G}$. We denote by the symbol $\int_{\mathcal{M}_{d}}$ the map from the equivariant cohomology of $\mathcal{M}_{d}$ to the equivariant cohomology of the point.

This shows how we should define the equivariant correlation functions. Let us choose $s$ generic points $x_{1}, \ldots, x_{s}$ on $\mathbf{C P}^{1}$. This gives us a map $\psi$ from $\mathcal{M}_{d}$ to $F^{s}$. For $s$ equivariant closed differential forms $p_{1}, p_{2}, \ldots, p_{s}$ (i.e. elements of our complex $C^{*}$ ) we denote by $P$ a product $P=\pi_{1}{ }^{*} p_{1} \wedge \pi_{2}{ }^{*} p_{2} \wedge \ldots \wedge \pi_{s}{ }^{*} p_{s}$.

Definition. The equivariant correlation function $\left\langle p_{1}\left|p_{2}\right| \ldots \mid p_{s}\right\rangle_{G}$ is defined by

$$
\left\langle p_{1}\left|p_{2}\right| \ldots \mid p_{s}\right\rangle_{G}=\sum_{d} q^{d} \int_{\mathcal{M}_{d}} \psi^{*}(P)
$$

By the same argument as in the case of the usual quantum cohomology, one can see that this definition is correct. The properties of the equivariant correlation functions are similar to those of the vertical correlation functions. Nevertheless we formulate them below.

\section{Properties of the equivariant correlation functions:}

1. They are multi-linear and skew-symmetric.

2. $\left\langle p_{1}\left|p_{2}\right| \ldots\left|p_{s}\right| 1\right\rangle_{G}=\left\langle p_{1}\left|p_{2}\right| \ldots \mid p_{s}\right\rangle_{G}$ ( where 1 is considered as a zero degree equivariant differential form ).

3. $\left.\left\langle p_{1}\left|p_{2}\right| \ldots \mid p_{s}\right\rangle_{G}\right|_{q=0}=\int\left(p_{1} \wedge \ldots \wedge p_{s}\right)$.

4. $\langle p\rangle_{G}=\int(p)$.

5. $\left\langle p_{1} \mid p_{2}\right\rangle_{(v)}=\int\left(p_{1} \wedge p_{2}\right)$.

6. Let $p$ be any element of $\left(S^{*}(g)^{*}\right)^{G}$. If we multiply $p$ by $1-$ a zero degree G-invariant differential form on $F$ - we can consider it as an element of the complex $C^{*}$. Then $\left\langle p \wedge p_{1}\left|p_{2}\right| \ldots \mid p_{s}\right\rangle_{G}=p\left\langle p_{1}\left|p_{2}\right| \ldots \mid p_{s}\right\rangle_{G}$ 
Let us explain a relation between the vertical correlation functions and the equivariant correlation functions. Suppose that we have an algebraic principal $G$-bundle $P$ over $B$. Assume that $P$ and $B$ are so nice that the vertical correlation functions are defined for $X=P \times_{G} F$ (that is, $X$ is a locally trivial algebraic bundle with the fiber $F$ over $B$ ). Let us choose a connection on $P$ represented by a 1 -form $\omega \in(\Omega(P, g))^{G}$. In this situation we have the following homomorphisms of differential graded algebras (see theorem 7.42 in 28])

$$
\begin{gathered}
\phi_{\omega}(X):\left(C^{*}, d_{g}\right) \rightarrow(\Omega(X), d) \\
\phi_{\omega}\left(\mathcal{M}_{d}\right):\left(\left(S^{*}(g)^{*} \otimes \Omega^{*}\left(\mathcal{M}_{d}\right)\right)^{G}, d_{g}\right) \rightarrow\left(\Omega\left(\mathcal{M}_{d}^{(v)}\right), d\right) \\
\phi_{\omega}:\left(\left(S^{*}(g)^{*}\right)^{G}, 0\right) \rightarrow(\Omega(B), d)
\end{gathered}
$$

These homomorphisms respect everything in the sense that if we take $s$ equivariant closed differential forms $p_{1}, p_{2}, \ldots, p_{s}$ on $F$, then the relation we are talking about is

$$
\phi_{\omega}\left(\left\langle p_{1}\left|p_{2}\right| \ldots \mid p_{s}\right\rangle_{G}\right)=\left\langle\phi_{\omega}(X)\left(p_{1}\right)\left|\phi_{\omega}(X)\left(p_{2}\right)\right| \ldots \mid \phi_{\text {omega }}(X)\left(p_{s}\right)\right\rangle_{(v)} .
$$

One can check this property using functoriality of the homomorphisms $\phi_{\omega}(X), \phi_{\omega}\left(\mathcal{M}_{d}\right)$, $\phi_{\omega}$ and others (see paragraph 7.6 in [28]).

Now we can define equivariant quantum cohomology. We have a spectral sequence $E_{2}^{p, q}=H^{p}\left(B G, H^{q}(F, \mathbf{K})\right.$ converging to the equivariant cohomology of $F$. Note that it degenerates in the term $E_{2}$ ( see [24]). So $H_{G}^{*}(F, \mathbf{K})$ is a free module over $H^{*}(B G, \mathbf{K})=$ $\left(S^{*}(g)^{*}\right)^{G} \otimes_{\mathbf{C}} \mathbf{K}$. The usual pairing $\int\left(p_{1} \wedge p_{2}\right)$ with values in $H^{*}(B G, \mathbf{K})$ is non-degenerate (since it respects the spectral sequence in natural way see [29]). We want to define a multiplication satisfying the following property:

$$
\left\langle p_{1} * p_{2} \mid p_{3}\right\rangle_{G}=\left\langle p_{1}\left|p_{2}\right| p_{3}\right\rangle_{G}
$$

Since $\left\langle p_{1} \mid p_{2}\right\rangle_{G}=\int\left(p_{1} \wedge p_{2}\right)$ we see that if the quantum multiplication exists, then it is unique. We can think of the triple $G$-equivariant pairing as a morphism of $H^{*}(B G, \mathbf{K})$ modules:

$$
H_{G}^{*}(F, \mathbf{K}) \otimes_{H^{*}(B G, \mathbf{K})} H_{G}^{*}(F, \mathbf{K}) \otimes_{H^{*}(B G, \mathbf{K})} H_{G}^{*}(F, \mathbf{K}) \rightarrow H^{*}(B G, \mathbf{K}) .
$$

Together with the non-degenerate Poincaré pairing, the triple pairing (4.3)defines a product

$$
H_{G}^{*}(F, \mathbf{K}) \otimes_{H^{*}(B G, \mathbf{K})} H_{G}^{*}(F, \mathbf{K}) \rightarrow H_{G}^{*}(F, \mathbf{K}) .
$$


We denote by $Q H_{G}^{*}(F, \mathbf{K})$ the algebra defined by this product.

One can check that this definition is correct and that the product (4.4)is associative. For example, associativity of the product can be proved by comparing (4.4)with the vertical quantum product ( using (4.2) ). One only needs to notice that there exists the a map from $Q H_{G}^{*}(F, \mathbf{K})$ to $Q H_{V}^{*}\left(P \times_{G} F, \mathbf{K}\right)$ which respects the multiplication.

So we have the equivariant quantum cohomology $\operatorname{ring} Q H_{G}^{*}(F, \mathbf{K})$. Moreover, $Q H_{G}^{*}(F, \mathbf{K})$ is an algebra over $H^{*}(B G, \mathbf{K})$. The following commutative diagram describes the relation between the equivariant and the vertical quantum cohomology:

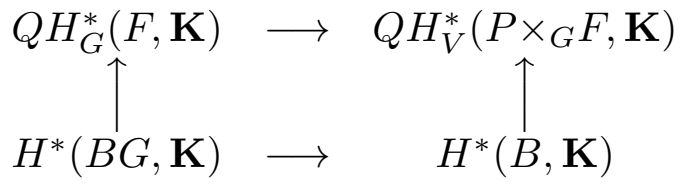

Now we discuss some properties of the equivariant quantum cohomology (see [1]).

Product. Let $F^{\prime}$ and $F^{\prime \prime}$ be $G^{\prime}$ - and $G^{\prime \prime}$-spaces respectively $\left(G^{\prime}, G^{\prime \prime}\right.$ are affine algebraic groups, etc. ). We have the cohomology groups $Q H_{G^{\prime}}^{*}\left(F^{\prime}, \mathbf{K}^{\prime}\right)$ and $Q H_{G^{\prime \prime}}^{*}\left(F^{\prime \prime}, \mathbf{K}^{\prime \prime}\right)$. Now take the product $F^{\prime} \times F^{\prime \prime}$ and consider it as a $G^{\prime} \times G^{\prime \prime}$-space. Consider the ring $Q H_{G^{\prime} \times G^{\prime \prime}}^{*}\left(F^{\prime} \times F^{\prime \prime}, \mathbf{K}^{\prime} \otimes_{\mathbf{C}} \mathbf{K}^{\prime \prime}\right)$. We can ask what is the relation between the equivariant quantum cohomology of $F^{\prime}, F^{\prime \prime}$ and that of $F^{\prime} \times F^{\prime \prime}$. The answer is given by the formula:

$$
Q H_{G^{\prime} \times G^{\prime \prime}}^{*}\left(F^{\prime} \times F^{\prime \prime}, \mathbf{K}^{\prime} \otimes_{\mathbf{C}} \mathbf{K}^{\prime \prime}\right)=Q H_{G^{\prime}}^{*}\left(F^{\prime}, \mathbf{K}^{\prime}\right) \otimes_{\mathbf{C}} Q H_{G^{\prime \prime}}^{*}\left(F^{\prime \prime}, \mathbf{K}^{\prime \prime}\right) .
$$

This formula follows immediately from the the definitions.

Restriction. Let $F$ be a $G$-space and suppose that we have an affine algebraic subgroup $\tilde{G}$ of $G$. Note that the Leray spectral sequence $E_{2}^{p, q}=H_{G}^{p}\left(p t, H^{q}(F, \mathbf{K})\right)\left(\tilde{E}_{2}^{p, q}=\right.$ $H_{\tilde{G}}^{p}\left(p t, H^{q}(F, \mathbf{K})\right)$ degenerates in the term $E_{2}$ ( respectively $\tilde{E}_{2}$ ). We have a natural morphism of the rings $f: H_{G}^{*}(F, \mathbf{K}) \rightarrow H_{\tilde{G}}^{*}(F, \mathbf{K})$ which comes from a map

$$
\left(S^{*}(g)^{*} \otimes \Omega^{*}(F)\right)^{G} \rightarrow\left(S^{*}(\tilde{g})^{*} \otimes \Omega^{*}(F)\right)^{\tilde{G}}
$$

induced by the inclusion $\tilde{g} \hookrightarrow g$. Let us make the following remark: $H_{\tilde{G}}^{*}(F, \mathbf{K})$ is generated by $H_{G}^{*}(F, \mathbf{K})$ as a $H_{\tilde{G}}^{*}(p t, \mathbf{K})$-module. Denote the natural map from $H_{G}^{*}(p t, \mathbf{K})$ to $H_{\tilde{G}}^{*}(p t, \mathbf{K})$ by $\delta$. If we look now at the definition of the equivariant correlation functions, we can easily see from functoriality ( paragraph 7.6 of [28] ) that

$$
\left\langle f\left(p_{1}\right)\left|f\left(p_{2}\right)\right| \ldots \mid f\left(p_{s}\right)\right\rangle_{\tilde{G}}=\delta\left(\left\langle p_{1}\left|p_{2}\right| \ldots \mid p_{s}\right\rangle_{G}\right) .
$$


Since the equivariant quantum product is uniquely defined by the correlation functions and because of the fact that $H_{\widetilde{G}}^{*}(F, \mathbf{K})$ is generated by $H_{G}^{*}(F, \mathbf{K})$ as a $H_{\widetilde{G}}^{*}(p t, \mathbf{K})$-module, the map $f$ respects the equivariant quantum product. So we have a morphism of rings:

$$
f: Q H_{G}^{*}(F, \mathbf{K}) \rightarrow Q H_{\tilde{G}}^{*}(F, \mathbf{K})
$$

Using the fact that the spectral sequence $\tilde{E}_{*}$ degenerates in $\tilde{E}_{2}$, we see that $Q H_{\tilde{G}}^{*}(F, \mathbf{K})$ is completely defined by $Q H_{G}^{*}(F, \mathbf{K}), H_{\tilde{G}}^{*}(F, \mathbf{K})$ and the morphism $f$. If $Q H_{G}^{*}(F, \mathbf{K})$ is equal to a quotient of an $H_{G}^{*}(p t, \mathbf{K})$-algebra $A$ modulo an ideal $I$, then $Q H_{\tilde{G}}^{*}(F, \mathbf{K})$ should be equal to the quotient of $A \otimes_{\mathbf{K}} H_{\tilde{G}}^{*}(p t, \mathbf{K})$ modulo the image of $I$.

Now we are going to discuss two particular cases of restriction.

1. Let $\tilde{G}$ be the trivial group. Then

$$
Q H_{\tilde{G}}^{*}(F, \mathbf{K})=Q H^{*}(F, \mathbf{K})
$$

So we see that the quantum cohomology of $F$ is equal to the quotient of the equivariant quantum cohomology modulo an ideal generated by the equivariant cohomology of the point. That is

$$
Q H^{*}(F, \mathbf{K})=Q H_{G}^{*}(F, \mathbf{K}) /\left(H_{G}^{*}(p t, \mathbf{K})\right)
$$

2. Let $H$ be any affine algebraic group, $K$ its maximal compact subgroup, and $G$ the corresponding reductive algebraic subgroup. Let $F$ be a $H$-space. Then $F$ naturally becomes a $G$-space. We claim that the following isomorphism takes place:

$$
Q H_{G}^{*}(F, \mathbf{K})=Q H_{H}^{*}(F, \mathbf{K})
$$

Really, we have $H_{H}^{*}(F, \mathbf{K})=H_{G}^{*}(F, \mathbf{K})$ ( since $F$ is a projective variety ) and $H_{H}^{*}(p t, *)=H_{G}^{*}(p t, *)$. From the restriction property we get

$$
Q H_{\widetilde{G}}^{*}(F, \mathbf{K})=Q H_{G}^{*}(F, \mathbf{K})
$$

as $H_{H}^{*}(p t, *)=H_{G}^{*}(p t, *)$-algebras.

2.1. Let $G$ be a parabolic subgroup of some semisimple group and $\tilde{G}$ be its Levi subgroup. There is a natural projection from $G$ onto $\tilde{G}$. Let $F$ be a $\tilde{G}$-space. Then $F$ naturally becomes a $G$-space. So we get ( see (4.8) )

$$
Q H_{G}^{*}(F, \mathbf{K})=Q H_{\tilde{G}}^{*}(F, \mathbf{K})
$$


Induction. Let $\tilde{G}$ be an affine algebraic subgroup of $G$ with a simply-connected compact Kähler quotient $G / \tilde{G}$. Let $F$ be a $\tilde{G}$-space and a simply-connected compact Kähler variety $X=G \times_{\tilde{G}} F$. One can wonder about the relations between $Q H_{\tilde{G}}^{*}(F, \tilde{\mathbf{K}})$ and $Q H_{G}^{*}(X, \mathbf{K})$. The answer is as follows.

First, the space $X$ is fibered over $G / \tilde{G}$ with a fiber $F$. We have a basis $\tilde{\omega}_{1}, \ldots, \tilde{\omega}_{k}$ in $H^{2}(F, \mathbf{Z}) \cap H^{1,1}(F, \mathbf{C})$. We can choose a basis $\omega_{1}, \ldots, \omega_{l}$ as we did in Section 3 when we described the vertical quantum cohomology. There exists a natural map from $\left(\left(S^{*}(g)^{*} \otimes \Omega(X)\right)^{G}, d_{g}\right)$ to $\left(\left(S^{*}(\tilde{g})^{*} \otimes \Omega(F)\right)^{\tilde{G}}, d_{\tilde{g}}\right)$ which induces an isomorphism of the equivariant cohomology.

Let us describe this map. We can treat $\left(S^{*}(g)^{*} \otimes \Omega(X)\right)^{G}$ as $\operatorname{Hom}_{G}\left(S^{*}(g), \Omega(X)\right)$. Indeed, if given a map $T$ then for any $v \in S^{*}(g)$ we have $T(v) \in \Omega(X)$. There is a canonical inclusion of $F$ into $X$ as a fiber over $1 \in(G / \tilde{G})$. So we can restrict the section $T(v)$ to $F$ :

$$
\left.\left.T(v)\right|_{F} \in \Omega(X)\right|_{F}=\Lambda^{*}(g / \tilde{g})^{*} \otimes_{\mathbf{C}} \Omega(F)
$$

To an element $T \in \operatorname{Hom}_{G}\left(S^{*}(g), \Omega(X)\right)$ we can associate an element

$$
\tilde{T} \in \operatorname{Hom}_{\tilde{G}}\left(S^{*}(g), \Lambda^{*}(g / \tilde{g})^{*} \otimes_{\mathbf{C}} \Omega(F)\right) .
$$

Thus we get a map from $\left(S^{*}(g)^{*} \otimes \Omega(X)\right)^{G}$ to $\left.\left(S^{*}(g)^{*} \otimes_{\mathbf{C}} \Lambda^{*}(g)^{*} / \tilde{g}\right) \otimes_{\mathbf{C}} \Omega(F)\right)^{\tilde{G}}$. There is a natural map from $S^{*}(g)^{*} \otimes_{\mathbf{C}} \Lambda^{*}(g / \tilde{g})^{*}$ to $S^{*}(\tilde{g})^{*}$ induced by inclusion of $S^{*}(\tilde{g})$ into $S^{*}(g) \otimes_{\mathbf{C}} \Lambda^{*}(g / \tilde{g})$. The composition of these two maps gives us the desired one. One can check that it respects the differential.

Using these natural maps, one can see that

$$
\left\langle p_{1}\left|p_{2}\right| \ldots \mid p_{s}\right\rangle_{\tilde{G}}=\left.\left\langle p_{1}\left|p_{2}\right| \ldots \mid p_{s}\right\rangle_{G}\right|_{q_{k+1}=0, \ldots, q_{l}=0}
$$

Finally, there is a natural isomorphism $H_{\tilde{G}}^{*}(F, *)=H_{G}^{*}(X, *)$, we obtain

$$
Q H_{\tilde{G}}^{*}(F, \tilde{\mathbf{K}})=\left.Q H_{G}^{*}(X, \mathbf{K})\right|_{q_{k+1}=0, \ldots, q_{l}=0}
$$

This is an isomorphism of the rings, but there are additional structures. The left hand side is an $H_{\tilde{G}}^{*}(p t, \tilde{\mathbf{K}})$-algebra and the right hand side is an $H_{G}^{*}(p t, \tilde{\mathbf{K}})$-algebra (since $\tilde{\mathbf{K}}=$ $\left.\mathbf{K} /\left(q_{k+1}, \ldots, q_{l}\right)\right)$. Let us describe the relation between these structures. One can see that there is a natural morphisms of the rings $H_{G}^{*}(p t, \tilde{\mathbf{K}}) \rightarrow H_{\tilde{G}}^{*}(p t, \tilde{\mathbf{K}})$. Thus the left hand side is also an $H_{G}^{*}(p t, \tilde{\mathbf{K}})$-algebra. One can see that isomorphism (4.10) is the isomorphism 
of $H_{G}^{*}(p t, \tilde{\mathbf{K}})$-algebras. relation We want to make a remark that this property of the equivariant quantum cohomology is an analog of the induction for the vertical quantum cohomology. One can prove this property reducing it to the same property of the vertical quantum cohomology.

Remark. The equivariant quantum cohomology gives us a simple way to compute the vertical quantum cohomology. If we had a principal $G$-bundle $P$ and a $G$-space $F$ then in order to compute the vertical quantum cohomology of $P \times{ }_{G} F$ it would be sufficient to know the equivariant quantum cohomology of $F$.

\section{Computation of the equivariant quantum cohomology of partial flag mani- folds.}

\subsection{Classical cohomology ring.}

Let us consider a partial flag manifold $F_{n_{1} \cdots n_{k}}$. There are $k$ canonical vector bundles $\xi_{1}, \ldots, \xi_{k}$ on this variety. To describe them we introduce $k$ auxiliary vector bundles $\eta_{1}, \ldots, \eta_{k}$. A fiber of $\eta_{i}$ over a flag $\left\{0 \subset V^{1} \subset \cdots \subset V^{k}\right\} \in F_{n_{1} \cdots n_{k}}$ is a vector space $V^{i}$. In particular, $\eta_{k}$ is a trivial bundle. The canonical bundles $\left\{\xi_{i}\right\}$ are defined by

$$
\xi_{i}= \begin{cases}\eta_{i} / \eta_{i-1}, & \text { if } i>1 \\ \eta_{1}, & \text { if } i=1\end{cases}
$$

We denote by $x_{j}^{(i)}$ the $j$-th Chern class of $\xi_{i}$.

The cohomology ring of the flag manifold is a quotient of the polynomial ring $\mathbf{C}\left[x_{1}^{(1)}, \ldots, x_{n_{k}}^{(k)}\right]$ modulo the ideal generated by the coefficients of the polynomial

$$
P(\lambda)=\lambda^{n}-\prod_{i=1}^{k}\left(\lambda^{n_{i}}+\lambda^{n_{i}-1} x_{1}^{(i)}+\cdots+x_{n_{i}}^{(i)}\right)
$$

We choose the basis $\omega_{1}=c_{1}\left(\eta_{1}\right), \ldots, \omega_{k-1}=c_{1}\left(\eta_{k-1}\right)$ in $H^{2}(X, \mathbf{Z}) \cap H^{1,1}(X, \mathbf{C})$.

Our partial flag manifold is a quotient $G L(n, \mathbf{C}) / P$ where $P$ is a corresponding parabolic subgroup. Let $\alpha_{1}, \ldots, \alpha_{n}$ be the simple positive roots of $\operatorname{sl}(n, \mathbf{C})$. To each $\alpha_{i}$ we can canonically associate a subgroup $S L_{(i)}(2, \mathbf{C}) \subset G L(n, \mathbf{C})$. If $i \neq n_{1}, i \neq n_{1}+n_{2}, \ldots$, $i \neq n_{1}+\ldots+n_{k-1}$ then $S L_{(i)}(2, \mathbf{C})$ lies in the parabolic subgroup $P$. On the other hand, if $i=n_{1}+\ldots+n_{j-1}$ then $S L_{(i)}(2, \mathbf{C})$ intersects $P$ along the maximal torus $\mathbf{C}^{*} \subset S L_{(i)}(2, \mathbf{C})$. So we get $k-1$ canonical embeddings of $\mathbf{C P}_{(i)}^{1}=S L_{(i)}(2, \mathbf{C}) / \mathbf{C}^{*}$ into $F_{n_{1} \cdots n_{k}}$. 
The homological type of curve $\Sigma \subset F_{n_{1} \cdots n_{k}}$ is given by the string $d=\left(\omega_{1}(\Sigma), \ldots, \omega_{k-1}(\Sigma)\right)$ of its coordinates. In particular, the homological type of $\mathbf{C P}_{\left(n_{1}+\cdots+n_{i}\right)}^{1}$ corresponding to the simple root $\alpha_{n_{1}+\cdots+n_{i}}$ is

$$
(\overbrace{0, \ldots, 0,1}^{i-1}, \overbrace{0, \ldots, 0}^{n-i})
$$

The group $G L(n, \mathbf{C})$ acts on the manifold $F_{n_{1} \cdots n_{k}}$. Let us describe the equivariant cohomology $H_{G L(n)}^{*}\left(F_{n_{1} \cdots n_{k}}, \mathbf{K}\right)$. As an algebra over $H_{G L(n)}^{*}(p t, \mathbf{K})=\mathbf{K}\left[c_{1}, \ldots, c_{n}\right]$ it is generated by the elements $\left\{y_{j}^{(i)}\right\}$. The map $H_{G L(n)}^{*}\left(F_{n_{1} \cdots n_{k}}, \mathbf{K}\right) \rightarrow H^{*}\left(F_{n_{1} \cdots n_{k}}, \mathbf{K}\right)$ sends $\left\{y_{j}^{(i)}\right\} \longrightarrow\left\{x_{j}^{(i)}\right\}$. The relations are given now by the coefficients of the polynomial

$$
P_{G L(n)}(\lambda)=\lambda^{n}+c_{1} \lambda^{n-1}+\ldots+c_{n}-\prod_{i=1}^{k}\left(\lambda^{n_{i}}+\lambda^{n_{i}-1} x_{1}^{(i)}+\cdots+x_{n_{i}}^{(i)}\right)
$$

\subsection{The equivariant quantum cohomology of Grassmanians}

The equivariant quantum cohomology ring $122 H_{G L(n)}^{*}(G r(n, m), \mathbf{K})$ of Grassmanian $G r(n, m)=U(n) /(U(n-m) \times U(m))$ as an algebra over $H_{G L(n)}^{*}(p t, \mathbf{K})$ is generated by $y^{(1)}, \ldots, y_{m}^{(1)}, y_{1}^{(2)}, \ldots, y_{n-m}^{(2)}$. By Lemma B2 (see Appendix B), the relations in $Q H_{G L(n)}(G r(n, m))$ are the deformations of the classical relations (5.4). In order to compute them we need to know the quantum cohomology ring $Q H^{*}(G r(n, m), \mathbf{K})$. It was discussed in many papers [12]- [17]. In the last reference it is shown that this ring is generated by $x^{(1)}, \ldots, x_{m}^{(1)}, x_{1}^{(2)}, \ldots, x_{n-m}^{(2)}$ and the relations are the coefficients of the following polynomial equation:

$$
\left(\lambda^{m}+\lambda^{m-1} x_{1}^{(1)}+\cdots+x_{m}^{(1)}\right)\left(\lambda^{n-m}+\lambda^{n-m-1} x_{1}^{(2)}+\cdots+x_{n-m}^{(2)}\right)=\lambda^{n}+(-1)^{n-m} q
$$

From Lemma B1 (see Appendix B) or [30] it follows that the whole ideal of relations in $Q H^{*}(G r(n, m), \mathbf{K})$ is generated by (5.5).

Now we can find the relations in $Q H_{G L(n)}^{*}(G r(n, m))$ we are after. Note that the degree of $q$ is equal to $2 n$. Therefore only the highest (2n) degree relation in (5.4)is deformed:

$$
y_{m}^{(1)} y_{n-m}^{(2)}=c_{n}+\alpha q
$$

where $\alpha$ is some constant. This constant is determined using the restriction property (see section 4). One can see it equals the corresponding constant $(-1)^{n-m}$ in (5.5). Note that this answer is consistent with the general formula (2.22) for the equivariant quantum cohomology of the partial flag manifolds.

12 For the Grassmanian the ring of coefficients is generated by a single parameter $q$ : $\mathbf{K}=\mathbf{C}[[q]]$. 


\subsection{The key lemma}

According to Lemma B2

$$
Q H_{G L(n)}^{*}\left(F_{n_{1} \cdots n_{k}}, \mathbf{K}\right)=\mathbf{K}\left[y_{1}^{(1)}, \ldots, y_{n_{k}}^{(k)} ; c_{1}, \ldots, c_{n}\right] / Q I_{G L(n)}
$$

where the ideal $Q I_{G L(n)}$ is generated by some deformations of the classical relations (5.4). The following Lemma is a trivial generalization of the Lemma 4.2.1 [1].

Lemma For $k>2$, suppose that a quasi-homogeneous relation of the form

$$
\lambda^{n}+c_{1} \lambda^{n-1}+\ldots+c_{n}-\prod_{i=1}^{k}\left(\lambda^{n_{i}}+\lambda^{n_{i}-1} x_{1}^{(i)}+\cdots+x_{n_{i}}^{(i)}\right)=O\left(q_{1}, \ldots, q_{k-1}\right)[\lambda, q, y, c]
$$

is satisfied in the equivariant quantum cohomology algebra of the partial flag manifold $F_{n_{1} \cdots n_{k}}$ modulo $q_{i}$ for each $i=1, \ldots, k-1$. Then the relation holds identically ( $i$. e. for all $q)$.

Sketch of the proof. Indeed, since the LHS of the relation in question is homogeneous of degree $2 n$ (we choose $\operatorname{deg} \lambda=2$ ), the hypothesis of Lemma 1 means that the difference $L H S-R H S$ is divisible by $q_{1} \ldots q_{k-1}$. But deg $q_{i}=n_{i}+n_{i+1}$ and

$$
\operatorname{deg} q_{1} \ldots q_{k-1}=2 n-n_{1}-n_{k}>n \text { for } k>2 \text {. }
$$

Q.E.D.

\subsection{Relations in $Q H_{G L(n)}^{*}\left(F_{n_{1} \cdots n_{k}}, \mathbf{K}\right)$}

We are going to prove that the relations (2.22) generate the ideal $Q I_{G L(n)}$. We will do this by induction on $k$.

1.) (A base of induction.) When $k=2$, the partial flag manifold $F_{n_{1} n_{2}}$ is called the Grassmanian $\operatorname{Gr}\left(n_{1}+n_{2}, n_{2}\right)$. We have already proven (2.22)in that case.

2.) (A step of induction.) Suppose that our assumption is true for all $k<k_{0}$. Let us prove it for $k=k_{0}$. According to the key lemma, it suffices to prove these relations modulo $q_{i}$ for all $i=1, \ldots k-1$.

Let us notice that 13

$$
F_{n_{1} \cdots n_{k}}=G L(n, \mathbf{C}) \times_{P_{j}}\left(F_{n_{1} \cdots n_{j}} \times F_{n_{j+1} \cdots n_{k}}\right)
$$

13 A symbol $F_{n}$ which may appear in this formula stands for a point $\{p t\}$. 
where $P_{j} \subset G L(n, \mathbf{C})$ is a corresponding parabolic subgroup. Using the induction property (4.10)we get

$$
\left.Q H_{G L(n, \mathbf{C})}^{*}\left(F_{n_{1} \cdots n_{k}}, \mathbf{K}\right)\right|_{q_{j}=0}=Q H_{P_{j}}^{*}\left(F_{n_{1} \cdots n_{j}} \times F_{n_{j+1} \cdots n_{k}}, \tilde{\mathbf{K}}\right)
$$

where $\tilde{\mathbf{K}}=\mathbf{K} /\left(\left(q_{j}\right)\right)$. From the restriction property (4.9) it follows that

$$
Q H_{P_{j}}^{*}\left(F_{n_{1} \cdots n_{j}} \times F_{n_{j+1} \cdots n_{k}}, \tilde{\mathbf{K}}\right)=Q H_{G L\left(N_{1}, \mathbf{C}\right) \times G L\left(N_{2}, \mathbf{C}\right)}^{*}\left(F_{n_{1} \cdots n_{j}} \times F_{n_{j+1} \cdots n_{k}}, \tilde{\mathbf{K}}\right)
$$

where $N_{1}=n_{1}+\ldots+n_{j}$ and $N_{2}=n_{j+1}+\ldots+n_{k}$. Finally, from the product (4.6)we have

$$
\begin{aligned}
Q H_{G L\left(N_{1}, \mathbf{C}\right) \times G L\left(N_{2}, \mathbf{C}\right)}^{*} & \left(F_{n_{1} \cdots n_{j}} \times F_{n_{j+1} \cdots n_{k}}, \tilde{\mathbf{K}}\right)= \\
= & Q H_{G L\left(N_{1}, \mathbf{C}\right)}^{*}\left(F_{n_{1} \cdots n_{j}}, \mathbf{K}_{1}\right) \otimes_{\mathbf{C}} Q H_{G L\left(N_{2}, \mathbf{C}\right)}^{*}\left(F_{n_{j+1} \cdots n_{k}}, \mathbf{K}_{2}\right)
\end{aligned}
$$

and $\tilde{\mathbf{K}}=\mathbf{K}_{1} \otimes \mathbf{K}_{2}$. Taking (5.8)-(5.10) together we obtain the natural isomorphism

$$
\left.Q H_{G L(n, \mathbf{C})}^{*}\left(F_{n_{1} \cdots n_{k}}, \mathbf{K}\right)\right|_{q_{j}=0}=Q H_{G L\left(N_{1}, \mathbf{C}\right)}^{*}\left(F_{n_{1} \cdots n_{j}}, \mathbf{K}_{1}\right) \otimes_{\mathbf{C}} Q H_{G L\left(N_{2}, \mathbf{C}\right)}^{*}\left(F_{n_{j+1} \cdots n_{k}}, \mathbf{K}_{2}\right) .
$$

We want to stress that (5.11) is an isomorphism of $Q H_{G L(n, \mathbf{C})}^{*}(p t, \tilde{\mathbf{K}})$-algebras. This isomorphism together with the assumption 2.) shows that for $k=k_{0}$ the relations (2.22) hold modulo $q_{j}$. Since it is true for all $j=1, \ldots, k-1$, the step of induction is complete.

Q.E.D.

Remark. Using the restriction property (4.7)we find that the quantum cohomology ring $Q H^{*}\left(F_{n_{1} \cdots n_{k}}, \mathbf{K}\right)$ is generated by $x_{1}^{(1)}, \ldots, x_{n_{k}}^{(k)}$ of degrees $\operatorname{deg} x_{i}^{(j)}=2 i$ with the relations given by the coefficients of the polynomial

$$
\operatorname{det}[\lambda \mathbf{1}+A]-\lambda^{n}
$$

where the $n \times n$ matrix $A$ is defined in the Section 2 just below the formula (2.22).

\section{Appendix A}

6.1. Remarks on the quantum cohomology $Q H^{*}(\mathbf{P}(E))$.

Whereas the vertical quantum cohomology of $F_{n_{1} \cdots n_{k}}(E)$ are described by (2.24), we do not now if it is possible to compute the quantum cohomology ring of the flag bundle in general. It seems to be quite a nontrivial problem, as two examples below show. 
Example 1. Take a projective space $\mathbf{P}^{m}$ as a base, a vector bundle $E=\oplus_{i=0}^{r_{i}} \mathcal{O}\left(r_{i}\right)$, $r_{0} \geq \cdots \geq r_{n}$ on it and let $X=\mathbf{P}(E)$. A Kähler cone of $\mathbf{P}(E)$ is two-dimensional and is generated by its two edges $y \in H^{11}\left(\mathbf{P}^{m}\right) \subset H^{11}(\mathbf{P}(E))$ and $z=x+r_{0} y$ where $x \in H^{11}(\mathbf{P}(E))$ is a generator as in (2.19) and $r_{0}=\max \left\{r_{i}\right\}$. The cohomology ring $H^{*}(\mathbf{P}(E))$ is generated by $x$ and $z$ with the relations

$$
\begin{aligned}
& y^{m+1}=0 \\
& \prod_{i=0}^{n}\left[z+\left(r_{i}-r_{0}\right) y\right]=0
\end{aligned}
$$

The first Chern class $c_{1}(\mathbf{P}(E))=n z+\left(m-\sum\left(r_{0}-r_{i}\right)\right) y$ is positive iff $k=m-\sum\left(r_{0}-r_{i}\right)>$ 0 . The Novikov ring $\mathbf{K}$ is generated by two elements (quantum deformation parameters) $q_{v}$ ("vertical") and $q_{h}$ ("horizontal") of degrees $2(n+1)$ and $2 k$ respectively.

The relations in the quantum cohomology ring $Q H^{*}(\mathbf{P}(E))$ in this example can be obtained using a fact $P(E)$ is a toric variety [9]. Equivalently, one finds the relations from the symplectic reduction of a linear $\sigma$-model on the $n+m+2$-dimensional vector space by the action of $U(1) \times U(1)[10]$. The quantum relations are:

$$
\begin{aligned}
& y^{m+1}=q_{h} \prod_{i=0}^{n}\left[z+\left(r_{i}-r_{0}\right) y\right]^{r_{0}-r_{i}} \\
& \prod_{i=0}^{n}\left[z+\left(r_{i}-r_{0}\right) y\right]=q_{v}
\end{aligned}
$$

Example 2. The quantum cohomology of the flag manifold $F_{3}=U(3) /(U(1) \times$ $U(1) \times U(1))$ are computed in [31, [1]. They are generated by two elements $x$ and $y$ of degree two. The edges of the two-dimensional Kähler cone of $F_{3}$ are $y$ and $x+y$. The quantum relations are:

$$
\begin{aligned}
& x^{2}+x y+y^{2}=q_{v}+q_{h} \\
& y^{3}=q_{h}(2 y+x)
\end{aligned}
$$

A space $F_{3}$ can be realized as a projectivization $\mathbf{P}(E)$ of a rank 2 irreducible vector bundle on $\mathbf{P}^{2}$, then $y$ is a generator of the ring $H^{*}\left(\mathbf{P}^{2}\right)$ and $x$ is a generator 4 as in (2.19). The first Chern class of $F_{3}$ is $c_{1}\left(F_{3}\right)=2(x+2 y)$ so the degrees of both $q_{v}$ and $q_{h}$ are 4 .

In both examples we see, that $Q H^{*}(\mathbf{P}(E))$ is not a $Q H^{*}(B)$-algebra. For $q_{h} \neq 0$ the relations for $y$ in (6.2), (6.3) differ from the relation in the quantum cohomology of

14 The Chern classes of $E$ are $c_{1}(E)=y$ and $c_{2}(E)=y^{2}$ 
projective space. This is a general situation in quantum cohomology; only in the classical limit "on the base" ( $q_{h}=0$ in our examples) does the "universality", like in (2.19), get restored and the ring $Q H^{*}(\mathbf{P}(E))$ becomes an algebra over $\left.Q H^{*}(B)\right|_{\text {class }}=H^{*}(B)$. But then it just coincides with the vertical quantum cohomology $Q H_{V}^{*}\left(\mathbf{P}(E), \mathbf{P}^{n}, B\right)$ as defined above.

It can happen sometime, like it happens in (6.2), that the "vertical" relation for $x$ is the same in both rings $Q H_{V}^{*}\left(\mathbf{P}(E), \mathbf{P}^{n}, B\right)$ and $Q H^{*}(\mathbf{P}(E))$. But the second example shows that in general this is not the case and in $Q H^{*}(\mathbf{P}(E))$ the "vertical" relations $Q H_{V}^{*}\left(\mathbf{P}(E), \mathbf{P}^{n}, B\right)$ get deformed by $q_{h}$ as well the "horizontal" relation do.

\section{Appendix B.}

We use the following setup. We have a compact Kähler algebraic variety $X ; c$ is the first Chern class of the tangent bundle to $X$. We denote by $q^{d}$ an element of the group ring of the sublattice $L$ in $H_{2}(X, \mathbf{Z}) \cap H_{2}(X, \mathbf{C})$ generated by the homology classes of rational complex curves $\left(\mathbf{C P}^{1}\right)$. Let us choose some basis $\omega_{1}, \ldots, \omega_{k}$ in $H^{2}(X, \mathbf{Z}) \cap H^{1,1}(X, \mathbf{C})$ such that the homology class of a curve $S$ is given by a string $d=\left(d_{1}, \ldots, d_{k}\right)$ of its coordinates with respect to $\omega_{1}, \ldots, \omega_{k}$ with $d_{1}, \ldots, d_{k} \geq 0$. Then the element $q^{d}$ can be identified with the monomial $q_{1}{ }^{d_{1}} \ldots q_{k} d_{k}$.

Let us define a ring of coefficients $\mathbf{K}=\mathbf{C}\left[\left[q_{1}, q_{2}, \ldots, q_{k}\right]\right]$. This is a local ring; let $I$ be its maximal ideal. The quantum cohomology is an algebra over the ring $\mathbf{K}$. Moreover, by definition it is a free module over $\mathbf{K}$. The ring $\mathbf{K}$ is graded so that $\operatorname{deg} q^{d}=c(d)$ (here we consider $d$ as an element of $H_{2}(X, \mathbf{C})$ ).

Let $p_{1}, \ldots, p_{l}$ be some cocycles of degree $r_{1}, \ldots, r_{l}$ respectively. Then a correlation function $\left\langle p_{1}\left|p_{2}\right| \ldots \mid p_{l}\right\rangle$ with values in $\mathbf{K}$ is defined. We assume that $\left\langle p_{1}\left|p_{2}\right| \ldots \mid p_{l}\right\rangle$ is always homogeneous of degree $r_{1}+r_{2}+\ldots+r_{l}-\operatorname{dim}(X)$. Notice that the pairing $\left\langle p_{1} \mid p_{2}\right\rangle$ is ordinary Poincaré pairing.

Let $x_{1}, \ldots, x_{r}$ be homogeneous generators of the ring $H^{*}(X, \mathbf{C})$. Let $f_{1}, \ldots, f_{s}$ be generators of the ideal of relations in $H^{*}(X, \mathbf{C})$ that is, $f_{1}, \ldots, f_{s}$ are homogeneous polynomials in $x_{1}, \ldots, x_{r}$ and $H^{*}(X, \mathbf{C})=S \Lambda\left[x_{1}, \ldots x_{r}\right] /\left(f_{1}, \ldots, f_{s}\right)$. Then the following lemma holds.

Lemma 1. There exist polynomials $g_{i}\left(x_{1}, \ldots, x_{r}, q_{1}, \ldots q_{k}\right)$ for $i=1, \ldots, s$ such that $g_{i}\left(x_{1}, \ldots x_{r}, 0, \ldots 0\right)=0 \quad i=1, \ldots, s$ with the following relations in $Q H^{*}(X, \mathbf{K})$

$$
f_{i}\left(x_{1}, \ldots, x_{r}\right)=g_{i}\left(x_{1}, \ldots, x_{r}, q_{1}, \ldots q_{k}\right)
$$


In this situation the quantum cohomology ring equals

$$
Q H^{*}(X, \mathbf{K})=S \Lambda_{\mathbf{K}}\left[x_{1}, \ldots x_{r}\right] /\left(f_{1}-g_{1}, \ldots, f_{s}-g_{s}\right)
$$

Proof: Let us choose some homogeneous basis $y_{1}, \ldots, y_{u}$ in the vector space $H^{*}(X, \mathbf{C})$. Let $z_{1}, \ldots, z_{u}$ be the dual basis with respect to Poincaré duality. Let $f_{i}\left(x_{1}, \ldots, x_{r}\right)=$ $\sum_{j_{1}, \ldots, j_{r}} a_{j_{1}, \ldots, j_{r}} x_{1}^{j_{1} \ldots x_{r}^{j_{r}}}$ and let $N$ be such positive integer that $a_{j_{1}, \ldots, j_{r}}=0$ for $j_{1}+\ldots+j_{r}>N$. Using the fact that $15\left\langle p_{1}\left|p_{2}\right| \ldots \mid p_{l}\right\rangle=\left\langle p_{1} * p_{2} * \ldots * p_{l-1} \mid p_{l}\right\rangle$ we can write

$$
\begin{gathered}
\sum_{j_{1}, \ldots, j_{r}} a_{j_{1}, \ldots, j_{r}}\langle\underbrace{x_{1}\left|x_{1}\right| \ldots \mid x_{1}}_{j_{1}}|\underbrace{x_{2}|\ldots| x_{2}}_{j_{2}}| \ldots|\underbrace{x_{r}|\ldots| x_{r}}_{j_{r}}| \underbrace{1|1| \ldots \mid 1}_{N-\left(j_{1}+\ldots+j_{r}\right)} \mid y_{j}\rangle= \\
=\left(f_{i}\left(x_{1}, \ldots, x_{r}\right), y_{j}\right)+h_{i, j}\left(q_{1}, \ldots, q_{k}\right)=h_{i, j}\left(q_{1}, \ldots, q_{k}\right),
\end{gathered}
$$

where $h_{i, j}\left(q_{1}, \ldots, q_{k}\right)$ is polynomial in $q_{1}, \ldots, q_{k}$ and $h_{i, j}(0, \ldots, 0)=0$.

So we see that in $Q H^{*}(X, \mathbf{K})$

$$
f_{i}\left(x_{1}, \ldots, x_{r}\right)=\sum_{j=1}^{u} h_{i, j}\left(q_{1}, \ldots, q_{k}\right) z_{j}
$$

Certainly we have a map from $S \Lambda\left[x_{1}, \ldots x_{r}\right]$ to $Q H^{*}(X, \mathbf{K})$ (as $\mathbf{K}$-modules). Moreover it is surjective since this map respects grading, the map from $S \Lambda\left[x_{1}, \ldots x_{r}\right] /(I)$ to $Q H^{*}(X, \mathbf{K}) /(I)$ is surjective and we can use Nakayama's lemma. So we can express $z_{j} \in Q H^{*}(X, \mathbf{K})$ as a polynomial of $x_{1}, \ldots, x_{r}$ with coefficients in $\mathbf{K}$. This proves the first part of the lemma.

We have a map from $S \Lambda\left[x_{1}, \ldots x_{r}\right] /\left(f_{1}-g_{1}, \ldots, f_{s}-g_{s}\right)$ to $Q H^{*}(X, \mathbf{K})$. These two $\mathbf{K}$-modules both are finitely generated and the isomorphism

$$
S \Lambda\left[x_{1}, \ldots x_{r}\right] /\left(f_{1}-g_{1}, \ldots, f_{s}-g_{s}\right) /(I)=Q H^{*}(X, \mathbf{K}) /(I)
$$

15 We can avoid using this fact since the quantum multiplication is a deformation of the ordinary one. We know that $x_{1}^{j_{1}} * \ldots * x_{r}^{j_{r}}=x_{1}^{j_{1}} \ldots x_{r}^{j_{r}}(\bmod I)$. So, instead of writing correlation functions, we can notice that $\left\langle x_{1}^{j_{1}} * \ldots * x_{r}^{j_{r}} \mid y_{j}\right\rangle=\left\langle x_{1}^{j_{1}} \ldots x_{r}^{j_{r}} \mid y_{j}\right\rangle$ ( $\left.\bmod I\right)$. As follows from the proof, knowing this we can avoid the formula $\left\langle p_{1}\left|p_{2}\right| \ldots \mid p_{l}\right\rangle=\left\langle p_{1} * p_{2} * \ldots * p_{l-1} \mid p_{l}\right\rangle$. 
holds. It follows now from the Nakayama's lemma that these modules are isomorphic:

$$
S \Lambda\left[x_{1}, \ldots x_{r}\right] /\left(f_{1}-g_{1}, \ldots, f_{s}-g_{s}\right)=Q H^{*}(X, \mathbf{K}) .
$$

Q.E.D.

This lemma shows that if we know the deformed relations $f_{i}=g_{i}$ then the quantum cohomology ring is $Q H^{*}(X, \mathbf{K})=S \Lambda_{\mathbf{K}}\left[x_{1}, \ldots x_{r}\right] /\left(f_{1}-g_{1}, \ldots, f_{s}-g_{s}\right)$.

Let us state the similar lemma for the equivariant quantum cohomology. We will not prove it since the proof is a simple adaptation of the proof of Lemma 1.

Let $\mathrm{G}$ be affine algebraic group acting on $X$. Let $y_{1}, \ldots, y_{r}$ be homogeneous generators of the algebra $H_{G}^{*}(X, \mathbf{C})$ over the ring $H_{G}^{*}(p t, \mathbf{C})$. Let $c_{1}, \ldots, c_{n}$ be some homogeneous generators of the ring $H_{G}^{*}(p t, \mathbf{C})$. Let $\bar{f}_{1}, \ldots, \bar{f}_{s}$ be generators of the ideal of relations between $y_{i}$. That is, $\bar{f}_{1}, \ldots, \bar{f}_{s}$ are homogeneous polynomials in $y_{1}, \ldots, y_{r}$ and $c_{1}, \ldots, c_{n}$ and

$$
H_{G}^{*}(X, \mathbf{C})=S \Lambda_{H_{G}^{*}(p t, \mathbf{C})}\left[y_{1}, \ldots, y_{r}\right] /\left(\bar{f}_{1}, \ldots, \bar{f}_{s}\right) .
$$

Lemma 2. There exist polynomials $\bar{g}_{i}\left(y_{1}, \ldots, y_{r}, c_{1}, \ldots, c_{n}, q_{1}, \ldots, q_{k}\right)$ for $i=1, \ldots, s$ such that $\bar{g}_{i}\left(y_{1}, \ldots, y_{r}, c_{1}, \ldots, c_{n}, 0, \ldots, 0\right)=0$ for $i=1, \ldots, s$ and the following relations in $Q H_{G}^{*}(X, \mathbf{K})$ hold

$$
\bar{f}_{i}\left(y_{1}, \ldots, y_{r}, c_{1}, \ldots, c_{n}\right)=\bar{g}_{i}\left(y_{1}, \ldots, y_{r}, c_{1}, \ldots, c_{n}, q_{1}, \ldots, q_{k}\right) .
$$

In this case the equivariant quantum cohomology ring

$$
Q H_{G}^{*}(X, \mathbf{K})=S \Lambda_{H_{G}^{*}(p t, \mathbf{K})}\left[y_{1}, \ldots, y_{r}\right] / v\left(\bar{f}_{1}-\bar{g}_{1}, \ldots, \bar{f}_{s}-\bar{g}_{s}\right)
$$

as $H_{G}^{*}(p t, \mathbf{K})$ algebra.

\section{Appendix C.}

We keep here the setup of appendix $\mathbf{B}$. Let us notice that the ring $\mathbf{K}$ ( the ring of "coefficients") depends on the choice of the basis in $H^{2}(X, \mathbf{Z}) \cap H^{1,1}(X, \mathbf{C})$. The reason is that the natural ring of "coefficients" $R$ is embedded into the $\operatorname{ring} \mathbf{K}$ (the ring $\mathbf{K}$ is bigger then the natural one). We use the ring $\mathbf{K}$ to simplify the explanations. Now we are going to describe the ring $R$. 
Let $H$ be a semigroup in $H_{2}(X, \mathbf{Z}) \cap H_{2}(X, \mathbf{C})$ generated by the homology classes of the rational complex curves $\left(\mathbf{C P}^{1}\right)$. Denote by $\tilde{R}=\mathbf{C}[H]$ the semigroup ring of $H$. There is a natural homomorphism from $\tilde{R}$ to $\mathbf{C}$ which sends $0 \in H$ to 1 and all other elements of $H$ to zero. We denote the kernel of this map by $J ; J$ is a maximal ideal. The ring $R$ is a completion of the ring $\tilde{R}$ with respect to the ideal $J$ :

$$
R=\hat{\tilde{R}}
$$

The quantum cohomology of $X\left(Q H^{*}(X, R)\right)$ is an algebra over $R$. One can see that there is a natural embedding of the ring $R$ into the $\mathbf{K}$ and that $Q H^{*}(X, \mathbf{K})=Q H^{*}(X, R) \otimes_{R} \mathbf{K}$. All the statements in this paper are true over the ring $R$ although they are formulated over the ring $\mathbf{K}$.

Moreover, if $c$ is positive then all our statements are true over the ring $\tilde{R}$ but we are not going to discuss that.

\section{Appendix D.}

We are going to show that the moduli spaces of algebraic maps of $\mathbf{C P}^{1}$ ( with three marked points ) to the projective variety $X$ exist.

1. For $X=\mathbf{C P}^{N}$ this fact is obvious.

2. This statement is also trivial for $X=\mathbf{P}^{N_{1}} \times \ldots \times \mathbf{P}^{N_{s}}$ since the moduli space of the product is the product of the moduli spaces.

3. For general $X$ let us choose an integral basis $\omega_{1}, \ldots, \omega_{s}$ of $H^{1,1}(X)$ such that $\omega_{i}$ belongs to the Kähler cone of $X$ for $i=1, \ldots, s$. Let $\mathcal{L}_{1}, \ldots, \mathcal{L}_{i}$ be the corresponding invertible sheaves. There exists $M \in \mathbf{N}$ such that all the sheaves $\mathcal{L}_{1}^{M}, \ldots, \mathcal{L}_{i}^{M}$ are very ample. Let us take the corresponding embeddigs $\sigma_{1}, \ldots, \sigma_{s}$ of $X$ into $\mathbf{P}^{N_{1}}, \ldots, \mathbf{P}^{N_{s}}$. Then the embedding $\Sigma=\sigma_{1} \times \ldots \times \sigma_{s}: X \hookrightarrow Z=\mathbf{P}^{N_{1}} \times \ldots \times \mathbf{P}^{N_{s}}$ has the following property: for any two algebraic curves $S_{1}$ and $S_{2}$ in $X$ such that $\left[S_{1}\right] \neq\left[S_{2}\right]\left(\left[S_{1}\right],\left[S_{2}\right] \in\right.$ $\left.H_{2}(X, \mathbf{C})\right)$ the pushforwards $\Sigma_{*}\left[S_{1}\right] \neq \Sigma_{*}\left[S_{2}\right]\left(\Sigma_{*}\left[S_{1}\right], \Sigma_{*}\left[S_{2}\right] \in H_{2}(Z, \mathbf{C})\right)$. Let $d \in H_{2}(X, \mathbf{C}) \cap H_{2}(X, \mathbf{Z})$. Denote by $\mathcal{M}_{\Sigma_{*} d}(Z)$ the moduili space of the agebraic maps $\mathbf{C P}^{1} \rightarrow Z$. An algebraic map $\mathbf{C P}^{1} \rightarrow X$ gives a point in $\mathcal{M}_{\Sigma_{*} d}(Z)$. Thus we can identify the space of algebraic maps $\mathbf{C P}^{1} \rightarrow Z$ as a subspace in $\mathcal{M}_{\Sigma_{*} d}(Z)$. One can see that the condition that the map $\phi: \mathbf{C P}^{1} \rightarrow Z$ factors through a map $\tilde{\phi}: \mathbf{C P}^{1} \rightarrow X$ so that $\phi=\Sigma \circ \tilde{\phi}$ is an algebraic condition. So the space $\mathcal{M}_{\Sigma_{*} d}(X)$ of algebraic maps $\mathbf{C P}^{1} \rightarrow X$ is an algebraic subvariety in $\mathcal{M}_{\Sigma_{*} d}(Z)$. 


\section{References}

[1] A. Givental, B. Kim Quantum Cohomology of Flag Manifolds and Toda Lattices preprint UC Berkeley, December 1993, hep-th 9312096.

[2] P. Candelas, P. Green, L. Parkes, X. de la Ossa A pair of Calabi-Yau manifolds as an exactly soluble superconformal field theory Nucl. Phys. B359 (1991) 21

[3] P. S. Aspinwall, B. R. Greene, D. R. Morrison Calabi-Yau Moduli Space, Mirror Manifolds and Spacetime Topology Change in String Theory., preprint IASSNS-HEP-93/38

[4] Albrecht Klemm and Stefan Theisen,Considerations of One-Modulus Calabi-Yau Compactifications: Picard-Fuchs Equations, Kähler Potentials and Mirror Maps, TUM-TH-143-92, KA-THEP-03/92

[5] Philip Candelas, Xenia de la Ossa, Anamaria Font, Sheldon Katz and David R. Morrison Mirror Symmetry for Two Parameter Models, preprint hep-th/9308083

[6] E. Witten Nucl. Phys. B340 (1990) 281

[7] A. Floer Symplectic Fixed Points and Holomorphic Spheres., Comm. Math. Phys. 120 (1989) 575-611

[8] M. Guest The Topology of the Space of Rational Curves on a Toric Variety, preprint alg-geom/9301005.

[9] V. Batyrev Quantum Cohomology Rings of Toric Manifolds, preprint 1993, alggeom/9310004

[10] E. Witten Phases of $N=2$ theories in two dimensions, preprint IASSNS-HEP-93/3

[11] S. Cecotti, C. Vafa On Classification of $N=2$ Sypersummetric Theories. preprint HUTP-92/A064

[12] D. Gepner, Fusion Rings And Geometry, Commun. Math. Phys. 141 (1991) 381

[13] D. Gepner and A. Schwimmer, Symplectic Fusion Rings And Their Metric, Nucl. Phys. B380 (1992) 147

[14] D. Gepner, Foundations Of Rational Conformal Field Theory, I (Cal Tech preprint, 1992).

[15] C. Vafa, Topological Mirrors And Quantum Rings, in Essays On Mirror Manifolds, ed. S.-T. Yau (International Press, 1992).

[16] K. Intriligator, Fusion Residues, Mod. Phys. Lett. A6 (1991) 3543.

[17] E. Witten The Verlinde Algebra And The Cohomology Of The Grassmannian, preprint IASSNS-HEP-93/41, hep-th/9312104

[18] A. Bertram, G. Daskalopolous, R. Wentworth Gromov Invariants for Holomorphic Maps from Riemann Surfaces to Grassmanians, Harvard preprint, April 1993

[19] L. Alvarez-Gaumé, P. Ginsparg Comm. Math. Phys. 102 (1985) 311

[20] W. Lerche, C. Vafa, N. Warner Chiral Rings in N=2 Superconformal Theory Nucl. Phys. B324 (1989) 427

[21] E. Witten Topological sigma model Comm. Math. Phys. 118(1988) 411 
[22] E. Witten Mirror manifolds and Topological Field Theory in Essays in Mirror Symmetry, ed. S. -T. Yau, 1992

[23] L. Bailieu, I. Singer The topological sigma model. Comm. Math. Phys. 125 (1989) 227

[24] P. Deligne Théorème de Lefschetz et critéres de dégénérescence de suite spectrales, Publ. Math. IHES, Vol 35(1968), pp. 107-126

[25] P.Griffits, J.Harris, Principles of algebraic geometry. Wiley, N.Y., 1978.

[26] R. Bott, F. Tu Differential Forms in Algebraic Topology

[27] G. Tian, S.-S. Roan, A mathematical theory of quantum cohomology, in preparation

[28] Nicole Berline, Ezra Getzler, Michele Vergne, Heat kernels and Dirac operators, Berlin; New York: Springer-Verlag, 1992, 369 p.

[29] V. Ginzburg, Equivariant cohomology and Kähler geometry Funct. Anal. Appl. 21:4 (1987), 271-283.

[30] S. Piunikhin, unpublished.

[31] V.Sadov, On equivalence of Floer's and quantum cohomology. Preprint HUTP93/A027. 